\title{
Dynamics of Mixed Dark Energy Domination in Teleparallel Gravity and Phase-Space Analysis
}

\author{
Emre Dil ${ }^{1}$ and Erdinç Kolay ${ }^{2}$ \\ ${ }^{1}$ Department of Physics, Sinop University, Korucuk, 57000 Sinop, Turkey \\ ${ }^{2}$ Department of Statistics, Sinop University, Korucuk, 57000 Sinop, Turkey \\ Correspondence should be addressed to Emre Dil; emredil@sakarya.edu.tr
}

Received 16 October 2015; Revised 26 November 2015; Accepted 29 November 2015

Academic Editor: Frank Filthaut

Copyright ( 2015 E. Dil and E. Kolay. This is an open access article distributed under the Creative Commons Attribution License, which permits unrestricted use, distribution, and reproduction in any medium, provided the original work is properly cited. The publication of this article was funded by $\mathrm{SCOAP}^{3}$.

\begin{abstract}
We consider a novel dark energy model to investigate whether it will provide an expanding universe phase. Here we propose a mixed dark energy domination which is constituted by tachyon, quintessence, and phantom scalar fields nonminimally coupled to gravity, in the absence of background dark matter and baryonic matter, in the framework of teleparallel gravity. We perform the phase-space analysis of the model by numerical methods and find the late-time accelerated attractor solutions implying the acceleration phase of universe.
\end{abstract}

\section{Introduction}

The universe is known to be experiencing an accelerating expansion by astrophysical observations such as Supernova Ia $[1,2]$, large scale structure $[3,4]$, the baryon acoustic oscillations [5], and cosmic microwave background radiation [6-9].

In order to explain the late-time accelerated expansion of universe, an unknown form of energy, called dark energy, is proposed. This unknown component of energy possesses some interesting properties; for instance, it is not clustered but spread all over the universe and its pressure is negative for driving the current acceleration of the universe. Observations show that the dark energy occupies $70 \%$ of our universe.

What is the constitution of the dark energy? One candidate for the answer of this question is the cosmological constant $\Lambda$ having a constant energy density filling the space homogeneously [10-13]. But cosmological constant is not well accepted since the cosmological problem [14] and the age problem [15]. For this reason, many other dark energy models have been proposed instead of the cosmological constant. Other candidates for dark energy constitution are quintessence, phantom, and tachyon fields. We can briefly classify the dark energy models in terms of the most powerful quantity of dark energy; its equation of state parameter $\omega_{\mathrm{DE}}=$ $p_{\mathrm{DE}} / \rho_{\mathrm{DE}}$, where $p_{\mathrm{DE}}$ and $\rho_{\mathrm{DE}}$ are the pressure and energy density of the dark energy, respectively. For cosmological constant boundary $\omega_{\mathrm{DE}}=-1$, but for quintessence the parameter $\omega_{\mathrm{DE}} \geq-1$, for phantom $\omega_{\mathrm{DE}} \leq-1$, and for nonminimally coupled tachyon with gravity both $\omega_{\mathrm{DE}} \leq-1$ and $\omega_{\mathrm{DE}} \geq-1[16-18]$.

The scenario of $\omega_{\mathrm{DE}}$ crossing the cosmological constant boundary is referred to as a "Quintom" scenario. The explicit construction of Quintom scenario has a difficulty, due to a no-go theorem. The equation of state parameter $\omega_{\mathrm{DE}}$ of a scalar field cannot cross the cosmological constant boundary according to no-go theorem, if the dark energy described by the scalar field is minimally coupled to gravity in FriedmannRobertson-Walker (FRW) geometry. The requirement for crossing the cosmological constant boundary is that the dark energy should be nonminimally coupled to gravity; namely, it should interact with the gravity [19-24]. There are also models in which possible coupling between dark energy and dark matter can occur $[25,26]$. In this paper, we consider a mixed dark energy model constituted by tachyon, quintessence, and phantom scalar fields nonminimally coupled to gravity.

The mixed dark energy model in this study is considered in the framework of teleparallel gravity instead of classical 
gravity. The teleparallel gravity is the equivalent form of the classical gravity, but in place of torsion-less Levi-Civita connection, curvature-less Weitzenbock one is used. The Lagrangian of teleparallel gravity contains torsion scalar $T$ constructed by the contraction of torsion tensor, in contrast to the Einstein-Hilbert action of classical gravity in which contraction of the curvature tensor $R$ is used. In teleparallel gravity, the dynamical variable is a set of four tetrad fields constructing the bases for the tangent space at each point of space-time [27-29]. The teleparallel gravity Lagrangian with only torsion scalar $T$ corresponds to the matter-dominated universe; namely, it does not accelerate. Therefore, to obtain a universe with an accelerating expansion, we can either replace $T$ with a function $f(T)$, the so-called $f(T)$ gravity (teleparallel analogue of $f(R)$ gravity) [30-32], or add an unknown form of energy, so-called dark energy, to the teleparallel gravity Lagrangian that allows also nonminimal coupling between dark energy and gravity to overcome the no-go theorem. The interesting feature of $f(T)$ theories is the existence of second or higher order derivatives in equations. Therefore, we prefer the second choice; adding extra scalar fields of the unknown energy forms as dark energy.

As different dark energy models, interacting teleparallel dark energy studies have been introduced in the literature; for instance, Geng et al. [33, 34] consider a quintessence scalar field with a nonminimal coupling between quintessence and gravity in the context of teleparallel gravity. The dynamics of this model has been studied in [35-37]. Tachyonic teleparallel dark energy is a generalization of the teleparallel quintessence dark energy by introducing a noncanonical tachyon scalar field in place of the canonical quintessence field [18, 38-40].

In this study, the main motivation is that we consider a more general dark energy model including three kinds of dark energy models, instead of taking one dark energy model as in $[16,18,38-40]$. In order to explain the expansion of universe by adding scalar fields as the dark energy constituents, there has never been assumed a cosmological model including three kinds of dark energy models. We assume tachyon, quintessence, and phantom fields as a mixed dark energy model which is nonminimally coupled to gravity in the framework of teleparallel gravity. We make the dynamical analysis of the model in FRW space-time. Later on, we translate the evolution equations into an autonomous dynamical system. After that the phase-space analysis of the model and the cosmological implications of the critical (or fixed) points of the model will be studied from the stability behavior of the critical points. Finally, we will make a brief summary of the results.

\section{Dynamics of the Model}

Our model consists of three scalar fields as the threecomponent dark energy domination without background dark matter and baryonic matter. These are the canonical scalar field, quintessence $\phi$, and two noncanonical scalar fields, tachyon $\psi$ and phantom $\sigma$, and all these scalar fields are nonminimally coupled to gravity. Since we consider only the dark energy dominated sector without the matter content of the universe, the action of the mixed teleparallel dark energy with a nonminimal coupling to the gravity can be written as $[16,38,39]$

$$
\begin{aligned}
S & =\int d^{4} x e\left[\frac{T}{2 \kappa^{2}}+\xi_{1} f(\psi) T\right. \\
& -V_{\psi}(\psi) \sqrt{1+g^{\mu \nu} \frac{\partial_{\mu} \psi \partial_{\nu} \psi}{V_{\psi}(\psi)}+\xi_{2} g(\phi) T} \\
& -\frac{1}{2} g^{\mu \nu} \partial_{\mu} \phi \partial_{\nu} \phi-V_{\phi}(\phi)+\xi_{3} h(\sigma) T+\frac{1}{2} g^{\mu \nu} \partial_{\mu} \sigma \partial_{\nu} \sigma \\
& \left.-V_{\sigma}(\sigma)\right]
\end{aligned}
$$

where $e=\operatorname{det}\left(e_{\mu}^{i}\right)=\sqrt{-g}$ and $e_{\mu}^{i}$ are the orthonormal tetrad components, such that

$$
g_{\mu \nu}=\eta_{i j} e_{\mu}^{i} e_{\nu}^{j},
$$

where $i, j$ run over $0,1,2$, and 3 for the tangent space at each point $x^{\mu}$ of the manifold and $\mu, \nu$ take the values $0,1,2$, and 3 and are the coordinate indices of the manifold. While $T$ is the torsion scalar, it is defined as

$$
T=\frac{1}{4} T^{\rho \mu \nu} T_{\rho \mu \nu}+\frac{1}{2} T^{\rho \mu \nu} T_{\nu \mu \rho}-T_{\mu \nu}^{\rho} T_{\rho}^{\nu \mu} .
$$

Here $T_{\mu \nu}^{\rho}$ is the torsion tensor constructed by the Weitzenbock connection $\Gamma_{\mu \nu}^{\rho}$, such that [41]

$$
T_{\mu \nu}^{\rho}=\Gamma_{\nu \mu}^{\rho}-\Gamma_{\mu \nu}^{\rho}=e_{i}^{\rho}\left(\partial_{\mu} e_{\nu}^{i}-\partial_{\nu} e_{\mu}^{i}\right)
$$

All the information about the gravitational field is contained in the torsion tensor $T_{\mu \nu}^{\rho}$ in teleparallel gravity. The Lagrangian of the theory is set up according to the conditions of invariance under general coordinate transformations, global Lorentz transformations, and the parity operations [42].

Furthermore, $\kappa^{2}=8 \pi G$ in (1) and $f(\psi), g(\phi)$, and $h(\sigma)$ are the functions responsible for nonminimal coupling between gravity $T$ and tachyon, quintessence, and phantom fields, respectively. $\xi_{1}, \xi_{2}$, and $\xi_{3}$ are the dimensionless coupling constants and $V_{\psi}(\psi), V_{\phi}(\phi)$, and $V_{\sigma}(\sigma)$ are the potentials for tachyon, quintessence, and phantom fields, respectively.

We consider a spatially flat FRW metric

$$
d s^{2}=d t^{2}-a^{2}(t)\left[d r^{2}+r^{2} d \Omega^{2}\right],
$$

and a tetrad field of the form $e_{\mu}^{i}=\operatorname{diag}(1, a, a, a)$. Then the Friedmann equations for FRW metric read as

$$
\begin{aligned}
H^{2} & =\frac{\kappa^{2}}{3}\left(\rho_{\psi}+\rho_{\phi}+\rho_{\sigma}\right), \\
\dot{H} & =-\frac{\kappa^{2}}{2}\left(\rho_{\psi}+p_{\psi}+\rho_{\phi}+p_{\phi}+\rho_{\sigma}+p_{\sigma}\right),
\end{aligned}
$$


where $H=\dot{a} / a$ is the Hubble parameter, $a$ is the scale factor, and dot represents the derivative with respect to cosmic time t. $\rho$ and $p$ are the energy density and the pressure of the corresponding scalar field constituents of the dark energy.

Conservation of energy gives the evolution equations for the dark energy constituents, such as

$$
\begin{gathered}
\dot{\rho}_{\psi}+3 H\left(\rho_{\psi}+p_{\psi}\right)=0 \\
\dot{\rho}_{\phi}+3 H\left(\rho_{\phi}+p_{\phi}\right)=0 \\
\dot{\rho}_{\sigma}+3 H\left(\rho_{\sigma}+p_{\sigma}\right)=0 .
\end{gathered}
$$

The total energy density and the pressure of dark energy read

$$
\begin{aligned}
& \rho_{\mathrm{tot}}=\rho_{\mathrm{DE}}=\rho_{\psi}+\rho_{\phi}+\rho_{\sigma}, \\
& p_{\mathrm{tot}}=p_{\mathrm{DE}}=p_{\psi}+p_{\phi}+p_{\sigma},
\end{aligned}
$$

with the total equation of state parameter

$$
\omega_{\text {tot }}=\frac{p_{\text {tot }}}{\rho_{\text {tot }}}=\omega_{\psi} \Omega_{\psi}+\omega_{\phi} \Omega_{\phi}+\omega_{\sigma} \Omega_{\sigma} \text {, }
$$

where $\omega_{\psi}=p_{\psi} / \rho_{\psi}, \omega_{\phi}=p_{\phi} / \rho_{\phi}$, and $\omega_{\sigma}=p_{\sigma} / \rho_{\sigma}$ are the equation of state parameters and $\Omega_{\psi}=\rho_{\psi} / \rho_{\text {tot }}, \Omega_{\phi}=\rho_{\phi} / \rho_{\text {tot }}$, and $\Omega_{\sigma}=\rho_{\sigma} / \rho_{\text {tot }}$ are the density parameters for the tachyon, quintessence, and phantom fields, respectively. Then the total density parameter is defined as

$$
\Omega_{\mathrm{tot}}=\Omega_{\psi}+\Omega_{\phi}+\Omega_{\sigma}=\frac{\kappa^{2} \rho_{\mathrm{tot}}}{3 H^{2}}=1
$$

where we assume that three kinds of scalar fields constitute the dark energy with an equal proportion of density parameter such that $\Omega_{\psi}=\Omega_{\phi}=\Omega_{\sigma}=1 / 3$.

The Lagrangian of the scalar fields is reexpressed from the action in (1), as

$$
\begin{aligned}
& L_{\psi}=\xi_{1} f(\psi) T-V_{\psi}(\psi) \sqrt{1-\frac{\dot{\psi}^{2}}{V_{\psi}(\psi)}}, \\
& L_{\phi}=\xi_{2} g(\phi) T+\frac{1}{2} \dot{\phi}^{2}-V_{\phi}(\phi), \\
& L_{\sigma}=\xi_{3} h(\sigma) T-\frac{1}{2} \dot{\sigma}^{2}-V_{\sigma}(\sigma) .
\end{aligned}
$$

Then the energy density and pressure values for three scalar fields can be found by the variation of the total Lagrangian in (1) with respect to the tetrad field $e_{i}^{\mu}$. After the variation of Lagrangian, there come contributions from the geometric terms so the $(0,0)$-component and $(i, i)$-component of the stress-energy tensor give the energy density and the pressure, respectively. Accordingly the energy density and pressure values for the tachyon, quintessence, and phantom fields read as

$$
\begin{aligned}
\rho_{\psi}= & -\xi_{1} f(\psi) 6 H^{2}+\frac{V_{\psi}(\psi)}{\sqrt{1-\left(\dot{\psi}^{2} / V_{\psi}(\psi)\right)}}, \\
p_{\psi}= & \xi_{1} f(\psi) 6 H^{2}-V_{\psi}(\psi) \sqrt{1-\frac{\dot{\psi}^{2}}{V_{\psi}(\psi)}} \\
& +4 \xi_{1} \dot{H} f(\psi)+4 \xi_{1} H f^{\prime}(\psi) \dot{\psi}^{2}, \\
\rho_{\phi}= & -\xi_{2} g(\phi) 6 H^{2}+\frac{1}{2} \dot{\phi}^{2}+V_{\phi}(\phi), \\
p_{\phi}= & \xi_{2} g(\phi) 6 H^{2}+\frac{1}{2} \dot{\phi}^{2}-V_{\phi}(\phi)+4 \xi_{2} \dot{H} g(\phi) \\
& +4 \xi_{2} H g^{\prime}(\phi) \dot{\phi}, \\
\rho_{\sigma}= & -\xi_{3} h(\sigma) 6 H^{2}-\frac{1}{2} \dot{\sigma}^{2}+V_{\sigma}(\sigma), \\
p_{\sigma}= & \xi_{3} h(\sigma) 6 H^{2}-\frac{1}{2} \dot{\sigma}^{2}-V_{\sigma}(\sigma)+4 \xi_{3} \dot{H} h(\sigma) \\
& +4 \xi_{3} H h^{\prime}(\sigma) \dot{\sigma},
\end{aligned}
$$

respectively. Here we have used the relation $T=-6 H^{2}$, and prime denotes the derivative of related coupling functions with respect to the related field variables. Now, we can find the equation of motions for three scalar fields from the variation of the field Lagrangians (11) with respect to the field variables $\psi, \phi$, and $\sigma$, such that

$$
\begin{aligned}
& \ddot{\psi}+3 H \dot{\psi}\left(1-\frac{\dot{\psi}^{2}}{V_{\psi}(\psi)}\right)+\left(1-\frac{3}{2} \frac{\dot{\psi}^{2}}{V_{\psi}(\psi)}\right) V_{\psi}(\psi) \\
& +6 \xi_{1}\left(1-\frac{\dot{\psi}^{2}}{V_{\psi}(\psi)}\right)^{3 / 2} H^{2} f^{\prime}(\psi)=0, \\
& \ddot{\phi}+V_{\phi}^{\prime}(\phi)+6 \xi_{2} H^{2} g^{\prime}(\phi)+3 H \dot{\phi}=0, \\
& \ddot{\sigma}-V_{\sigma}^{\prime}(\sigma)-6 \xi_{3} H^{2} h^{\prime}(\sigma)+3 H \dot{\sigma}=0 .
\end{aligned}
$$

These equations of motions are for the tachyon, quintessence, and phantom constituents of dark energy, respectively. Here, the prime of potentials denotes the derivative of related field potentials with respect to the related field variables. All these evolution equations in (18) can also be obtained by using the relations (12)-(17) in the continuity equations (7).

We now perform the phase-space analysis of the model in order to investigate the late-time solutions of the universe considered here.

\section{Phase-Space and Stability Analysis}

We study the properties of the constructed dark energy model by performing the phase-space analysis. Therefore we transform the aforementioned dynamical system into its 
autonomous form $[38,39,43-46]$. To proceed we introduce the auxiliary variables

$$
\begin{aligned}
& x_{\psi}=\frac{\dot{\psi}}{\sqrt{V_{\psi}(\psi)}}, \\
& y_{\psi}=\frac{\kappa \sqrt{V_{\psi}(\psi)}}{\sqrt{3} H}, \\
& u_{\psi}=\kappa \sqrt{f(\psi)}, \\
& x_{\phi}=\frac{\kappa \dot{\phi}}{\sqrt{6} H}, \\
& y_{\phi}=\frac{\kappa \sqrt{V_{\phi}(\phi)}}{\sqrt{3} H}, \\
& u_{\phi}=\kappa \sqrt{g(\phi)}, \\
& u_{\sigma}=\kappa \sqrt{h(\sigma)}, \\
& x_{\sigma}=\frac{\kappa \sqrt{V_{\sigma}(\sigma)}}{\sqrt{6} H},
\end{aligned}
$$

together with $N=\ln a$ and for any quantity $F$, the time derivative is $\dot{F}=H(d F / d N)$.

We rewrite the density parameters for the fields $\psi, \phi$, and $\sigma$ in the autonomous system by using (10), (12), (14), and (16) with (19)

$$
\begin{aligned}
& \Omega_{\psi}=\frac{\kappa^{2} \rho_{\psi}}{3 H^{2}}=\mu y_{\psi}^{2}-2 \xi_{1} u_{\psi}^{2}, \\
& \Omega_{\phi}=\frac{\kappa^{2} \rho_{\phi}}{3 H^{2}}=x_{\phi}^{2}+y_{\phi}^{2}-2 \xi_{2} u_{\phi}^{2}, \\
& \Omega_{\sigma}=\frac{\kappa^{2} \rho_{\sigma}}{3 H^{2}}=-x_{\sigma}^{2}+y_{\sigma}^{2}-2 \xi_{3} u_{\sigma}^{2},
\end{aligned}
$$

and the total density parameter is

$$
\begin{aligned}
\Omega_{\mathrm{tot}}= & \frac{\kappa^{2} \rho_{\mathrm{tot}}}{3 H^{2}}=\Omega_{\psi}+\Omega_{\phi}+\Omega_{\sigma} \\
= & \mu y_{\psi}^{2}-2 \xi_{1} u_{\psi}^{2}+x_{\phi}^{2}+y_{\phi}^{2}-2 \xi_{2} u_{\phi}^{2}-x_{\sigma}^{2}+y_{\sigma}^{2} \\
& -2 \xi_{3} u_{\sigma}^{2}=1,
\end{aligned}
$$

where $\mu=1 / \sqrt{1-\left(\dot{\psi}^{2} / V_{\psi}(\psi)\right)}=1 / \sqrt{1-x_{\psi}^{2}}$. Then the equation of state parameters can be written in the autonomous form by using (12) $-(17)$ in $\omega=p / \rho$ for every scalar field, such as

$$
\begin{aligned}
& \omega_{\psi}=\frac{p_{\psi}}{\rho_{\psi}} \\
& =\frac{-\mu^{-1} y_{\psi}^{2}+2 \xi_{1} u_{\psi}\left[(2 \sqrt{3} / 3) \alpha_{\psi} x_{\psi} y_{\psi}+u_{\psi}(1-(2 / 3) s)\right]}{\mu y_{\psi}^{2}-2 \xi_{1} u_{\psi}^{2}}, \\
& \omega_{\phi}=\frac{p_{\phi}}{\rho_{\phi}} \\
& =\frac{x_{\phi}^{2}-y_{\phi}^{2}+2 \xi_{2} u_{\phi}\left[(2 \sqrt{6} / 3) \alpha_{\phi} x_{\phi}+u_{\phi}(1-(2 / 3) s)\right]}{x_{\phi}^{2}+y_{\phi}^{2}-2 \xi_{2} u_{\phi}^{2}}, \\
& \omega_{\sigma}=\frac{p_{\sigma}}{\rho_{\sigma}} \\
& =\frac{-x_{\sigma}^{2}-y_{\sigma}^{2}+2 \xi_{3} u_{\sigma}\left[(2 \sqrt{6} / 3) \alpha_{\sigma} x_{\sigma}+u_{\sigma}(1-(2 / 3) s)\right]}{-x_{\sigma}^{2}+y_{\sigma}^{2}-2 \xi_{3} u_{\sigma}^{2}},
\end{aligned}
$$

where $\alpha_{\psi}=f^{\prime}(\psi) / \sqrt{f(\psi)}, \alpha_{\phi}=g^{\prime}(\phi) / \sqrt{g(\phi)}, \alpha_{\sigma}=$ $h^{\prime}(\sigma) / \sqrt{h(\sigma)}$ and $s=-\dot{H} / H^{2}$. From (9) and (20) and (22), we obtain $\omega_{\text {tot }}$ in the autonomous system, as

$$
\begin{aligned}
\omega_{\text {tot }}= & -\mu^{-1} y_{\psi}^{2} \\
& +2 \xi_{1} u_{\psi}\left[\frac{2 \sqrt{3}}{3} \alpha_{\psi} x_{\psi} y_{\psi}+u_{\psi}\left(1-\frac{2}{3} s\right)\right]+x_{\phi}^{2} \\
& -y_{\phi}^{2}+2 \xi_{2} u_{\phi}\left[\frac{2 \sqrt{6}}{3} \alpha_{\phi} x_{\phi}+u_{\phi}\left(1-\frac{2}{3} s\right)\right] \\
& -x_{\sigma}^{2}-y_{\sigma}^{2} \\
& +2 \xi_{3} u_{\sigma}\left[\frac{2 \sqrt{6}}{3} \alpha_{\sigma} x_{\sigma}+u_{\sigma}\left(1-\frac{2}{3} s\right)\right]
\end{aligned}
$$

We can express $s$ in the autonomous system by using (6) and (23), such that

$$
\begin{aligned}
s= & -\frac{\dot{H}}{H^{2}}=\frac{3}{2}\left(1+\omega_{\text {tot }}\right)=\frac{3}{2}-\frac{3}{2} \mu^{-1} y_{\psi}^{2} \\
& +\xi_{1} u_{\psi}\left[2 \sqrt{3} \alpha_{\psi} x_{\psi} y_{\psi}+u_{\psi}(3-2 s)\right]+\frac{3}{2} x_{\phi}^{2}-\frac{3}{2} y_{\phi}^{2} \\
& +\xi_{2} u_{\phi}\left[2 \sqrt{6} \alpha_{\phi} x_{\phi}+u_{\phi}(3-2 s)\right]-\frac{3}{2} x_{\sigma}^{2}-\frac{3}{2} y_{\sigma}^{2} \\
& +\xi_{3} u_{\sigma}\left[2 \sqrt{6} \alpha_{\sigma} x_{\sigma}+u_{\sigma}(3-2 s)\right], \\
s= & \left\{\frac{3}{2}-\frac{3}{2} \mu^{-1} y_{\psi}^{2}+\xi_{1} u_{\psi}\left[2 \sqrt{3} \alpha_{\psi} x_{\psi} y_{\psi}+3 u_{\psi}\right]\right. \\
& +\frac{3}{2} x_{\phi}^{2}-\frac{3}{2} y_{\phi}^{2}+\xi_{2} u_{\phi}\left[2 \sqrt{6} \alpha_{\phi} x_{\phi}+3 u_{\phi}\right]-\frac{3}{2} x_{\sigma}^{2} \\
& \left.-\frac{3}{2} y_{\sigma}^{2}+\xi_{3} u_{\sigma}\left[2 \sqrt{6} \alpha_{\sigma} x_{\sigma}+3 u_{\sigma}\right]\right\}\left[1+2 \xi_{1} u_{\psi}^{2}\right. \\
& \left.+2 \xi_{2} u_{\phi}^{2}+2 \xi_{3} u_{\sigma}^{2}\right]^{-1} .
\end{aligned}
$$


Here $s$ is only a jerk parameter used in other equations of cosmological parameters. Then the deceleration parameter $q$ is

$$
\begin{aligned}
q= & -1-\frac{\dot{H}}{H^{2}}=\frac{1}{2}+\frac{3}{2} \omega_{\text {tot }} \\
= & \frac{1}{2}-\frac{3}{2} \mu^{-1} y_{\psi}^{2}+\xi_{1} u_{\psi}\left[2 \sqrt{3} \alpha_{\psi} x_{\psi} y_{\psi}+u_{\psi}(3-2 s)\right] \\
& +\frac{3}{2} x_{\phi}^{2}-\frac{3}{2} y_{\phi}^{2}+\xi_{2} u_{\phi}\left[2 \sqrt{6} \alpha_{\phi} x_{\phi}+u_{\phi}(3-2 s)\right] \\
& -\frac{3}{2} x_{\sigma}^{2}-\frac{3}{2} y_{\sigma}^{2}+\xi_{3} u_{\sigma}\left[2 \sqrt{6} \alpha_{\sigma} x_{\sigma}+u_{\sigma}(3-2 s)\right]
\end{aligned}
$$

Now we transform the equations of motions (6) and (18) into the autonomous system containing the auxiliary variables in (19) and their derivatives with respect to $N=\ln a$. Thus we obtain $X^{\prime}=f(X)$, where $X$ is the column vector including the auxiliary variables and $f(X)$ is the column vector of the autonomous equations. After writing $X^{\prime}$, we find the critical points $X_{c}$ of $X$, by setting $X^{\prime}=0$. We expand $X^{\prime}=f(X)$ around $X=X_{c}+U$, where $U$ is the column vector of perturbations of the auxiliary variables, such as $\delta x, \delta y$, and $\delta u$ for each scalar field. Thus, we expand the perturbation equations up to the first order for each critical point as $U^{\prime}=$ $M U$, where $M$ is the matrix of perturbation equations. For each critical point, the eigenvalues of perturbation matrix $M$ determine the type and stability of the critical points [47-50].

Particularly, the autonomous form of the cosmological system in (6) and (18) is [51-60]

$$
\begin{aligned}
x_{\psi}^{\prime} & =\frac{\sqrt{3}}{2}\left[\lambda_{\psi} x_{\psi}^{2} y_{\psi}+\lambda_{\psi}\left(2-3 x_{\psi}^{2}\right) y_{\psi}\right. \\
& \left.-4 \alpha_{\psi} \xi_{1} u_{\psi} \mu^{-3} y_{\psi}^{-1}-2 \sqrt{3} x_{\psi} \mu^{-2}\right], \\
y_{\psi}^{\prime}= & {\left[-\frac{\sqrt{3}}{2} \lambda_{\psi} x_{\psi} y_{\psi}+s\right] y_{\psi}, } \\
u_{\psi}^{\prime}= & \frac{\sqrt{3}}{2} \alpha_{\psi} x_{\psi} y_{\psi}, \\
x_{\phi}^{\prime}= & -3 x_{\phi}\left[1+x_{\sigma}^{2}-x_{\phi}^{2}+\frac{2}{3} \xi_{2}\left(s u_{\phi}-\sqrt{6} \alpha_{\phi} x_{\phi}\right) u_{\phi}\right. \\
& +\frac{2}{3} \xi_{3}\left(s u_{\sigma}-\sqrt{6} \alpha_{\sigma} x_{\sigma}\right) u_{\sigma}-\frac{\mu}{2} x_{\psi}^{2} y_{\psi}^{2} \\
& \left.+\frac{2}{3} \xi_{1}\left(s u_{\psi}-\sqrt{6} \alpha_{\psi} x_{\psi}\right) u_{\psi}\right]+\lambda_{\phi} \frac{\sqrt{6}}{2} y_{\phi}^{2} \\
& -\sqrt{6} \xi_{2} \alpha_{\phi} u_{\phi}, \\
& \left.-\frac{2}{3} \xi_{3}\left(s u_{\sigma}-\sqrt{6} \alpha_{\sigma} x_{\sigma}\right) u_{\sigma}-\lambda_{\phi} \frac{\sqrt{6}}{6} x_{\phi}^{2}\right], \\
y_{\phi}^{\prime} & =3 y_{\phi}\left[\frac{\mu}{2} x_{\psi}^{2} y_{\psi}^{2}-\frac{2}{3} \xi_{1}\left(s u_{\psi}-\sqrt{6} \alpha_{\psi} x_{\psi}\right) u_{\psi}+x_{\phi}^{2}\right. \\
& \left.2 s \alpha_{\phi} x_{\phi}\right) u_{\phi}-x_{\sigma}^{2}
\end{aligned}
$$

$$
\begin{aligned}
u_{\phi}^{\prime} & =\frac{\sqrt{6}}{2} \alpha_{\phi} x_{\phi}, \\
x_{\sigma}^{\prime} & =-3 x_{\sigma}\left[1+x_{\sigma}^{2}-x_{\phi}^{2}+\frac{2}{3} \xi_{2}\left(s u_{\phi}-\sqrt{6} \alpha_{\phi} x_{\phi}\right) u_{\phi}\right. \\
& +\frac{2}{3} \xi_{3}\left(s u_{\sigma}-\sqrt{6} \alpha_{\sigma} x_{\sigma}\right) u_{\sigma}-\frac{\mu}{2} x_{\psi}^{2} y_{\psi}^{2} \\
& \left.+\frac{2}{3} \xi_{1}\left(s u_{\psi}-\sqrt{6} \alpha_{\psi} x_{\psi}\right) u_{\psi}\right]+\lambda_{\sigma} \frac{\sqrt{6}}{2} y_{\sigma}^{2} \\
& +\sqrt{6} \xi_{3} \alpha_{\sigma} u_{\sigma}, \\
y_{\sigma}^{\prime} & =3 y_{\sigma}\left[\frac{\mu}{2} x_{\psi}^{2} y_{\psi}^{2}-\frac{2}{3} \xi_{1}\left(s u_{\psi}-\sqrt{6} \alpha_{\psi} x_{\psi}\right) u_{\psi}+x_{\phi}^{2}\right. \\
& -\frac{2}{3} \xi_{2}\left(s u_{\phi}-\sqrt{6} \alpha_{\phi} x_{\phi}\right) u_{\phi}-x_{\sigma}^{2} \\
& \left.-\frac{2}{3} \xi_{3}\left(s u_{\sigma}-\sqrt{6} \alpha_{\sigma} x_{\sigma}\right) u_{\sigma}-\lambda_{\sigma} \frac{\sqrt{6}}{6} x_{\sigma}^{2}\right] \\
u_{\sigma}^{\prime}= & \frac{\sqrt{6}}{2} \alpha_{\sigma} x_{\sigma},
\end{aligned}
$$

where $\lambda_{\psi}=-V_{\psi}^{\prime} / \kappa V_{\psi}, \lambda_{\phi}=-V_{\phi}^{\prime} / \kappa V_{\phi}$, and $\lambda_{\sigma}=-V_{\sigma}^{\prime} / \kappa V_{\sigma}$. Henceforth, we assume the nonminimal coupling functions $f(\psi) \propto \psi^{2}, g(\phi) \propto \phi^{2}$, and $h(\sigma) \propto \sigma^{2}$; thus $\alpha_{\psi}, \alpha_{\phi}$, and $\alpha_{\sigma}$ are constant. Also the usual assumption in the literature is to take the potentials $V_{\psi}=V_{\psi 0} e^{-k_{\psi} \lambda_{\psi} \psi}, V_{\phi}=V_{\phi 0} e^{-k_{\phi} \lambda_{\phi} \phi}$, and $V_{\sigma}=V_{\sigma 0} e^{-k_{\sigma} \lambda_{\sigma} \sigma}[43,61-63]$. Such potentials give also constants $\lambda_{\psi}, \lambda_{\phi}$, and $\lambda_{\sigma}$.

Now we perform the phase-space analysis of the model by finding the critical points of the autonomous system in (26). To obtain these points, we set the left hand sides of (26) to zero. After some calculations, four critical points are found by assuming $\omega_{\text {tot }}$ and $q$ as -1 for each critical point. The critical points are listed in Table 1 with the existence conditions.

Then we insert linear perturbations $x \rightarrow x_{c}+\delta x, y \rightarrow$ $y_{c}+\delta y$, and $u \rightarrow u_{c}+\delta u$ about the critical points for three scalar fields $\psi, \phi$, and $\sigma$ in the autonomous system (26). Thus we obtain a $9 \times 9$ perturbation matrix $M$ whose elements are given as

$$
\begin{aligned}
& M_{11}=-3, \\
& M_{12}=\sqrt{3} \lambda_{\psi}+2 \sqrt{3} \alpha_{\psi} \xi_{1} y_{\psi}^{-2} u_{\psi}, \\
& M_{13}=-2 \sqrt{3} \alpha_{\psi} \xi_{1} y_{\psi}^{-1}, \\
& M_{21}=\frac{2 \sqrt{3} \alpha_{\psi} \xi_{1}}{P} u_{\psi} y_{\psi}^{2}-\frac{\sqrt{3}}{2} \lambda_{\psi} y_{\psi}^{2}, \\
& M_{22}=-\frac{3}{P} y_{\psi}^{2}, \\
& M_{23}=\frac{6 \xi_{1}}{P} u_{\psi} y_{\psi}, \\
& M_{24}=\frac{2 \sqrt{6} \alpha_{\psi} \xi_{2}}{P} u_{\phi} y_{\psi},
\end{aligned}
$$



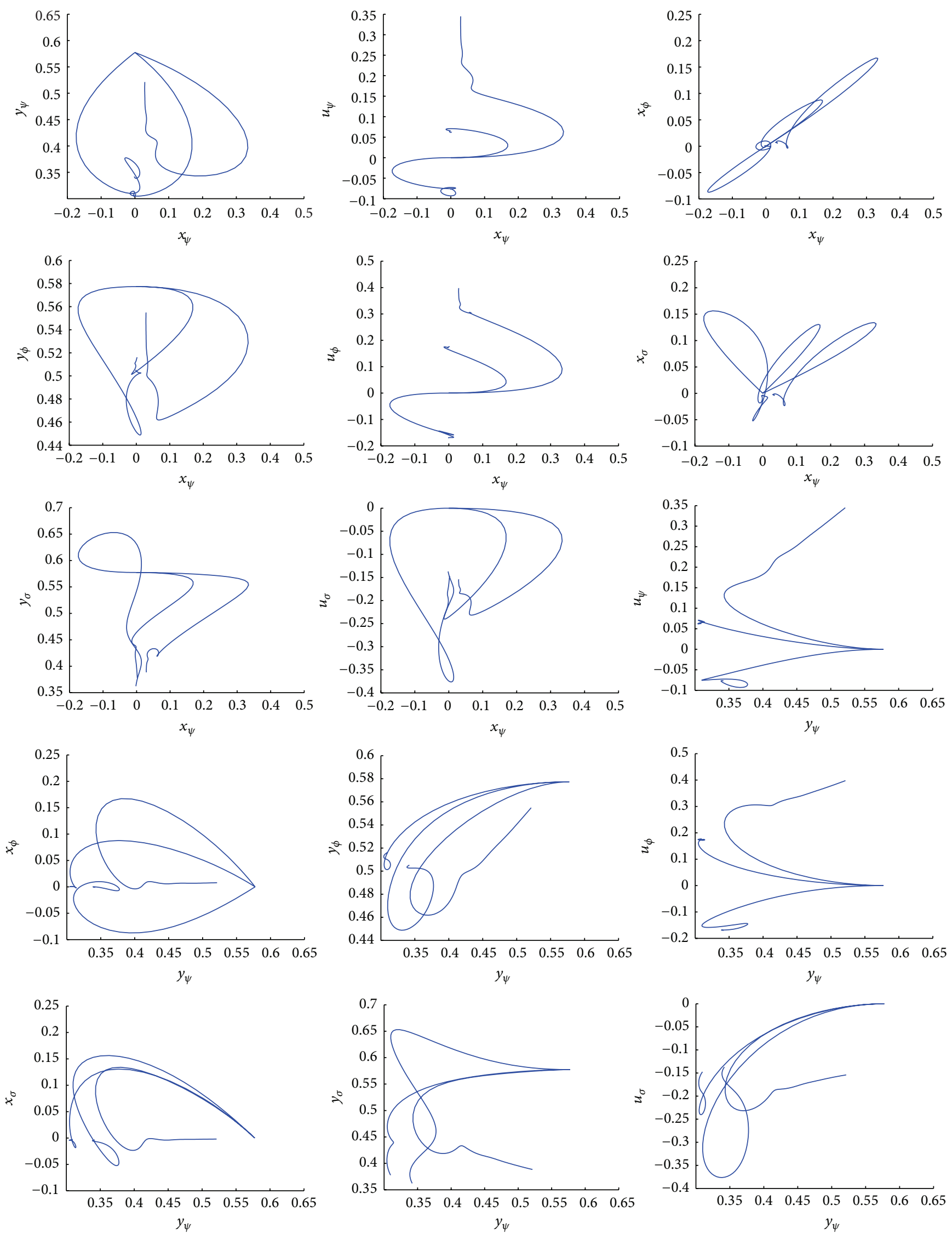

Figure 1: Continued. 

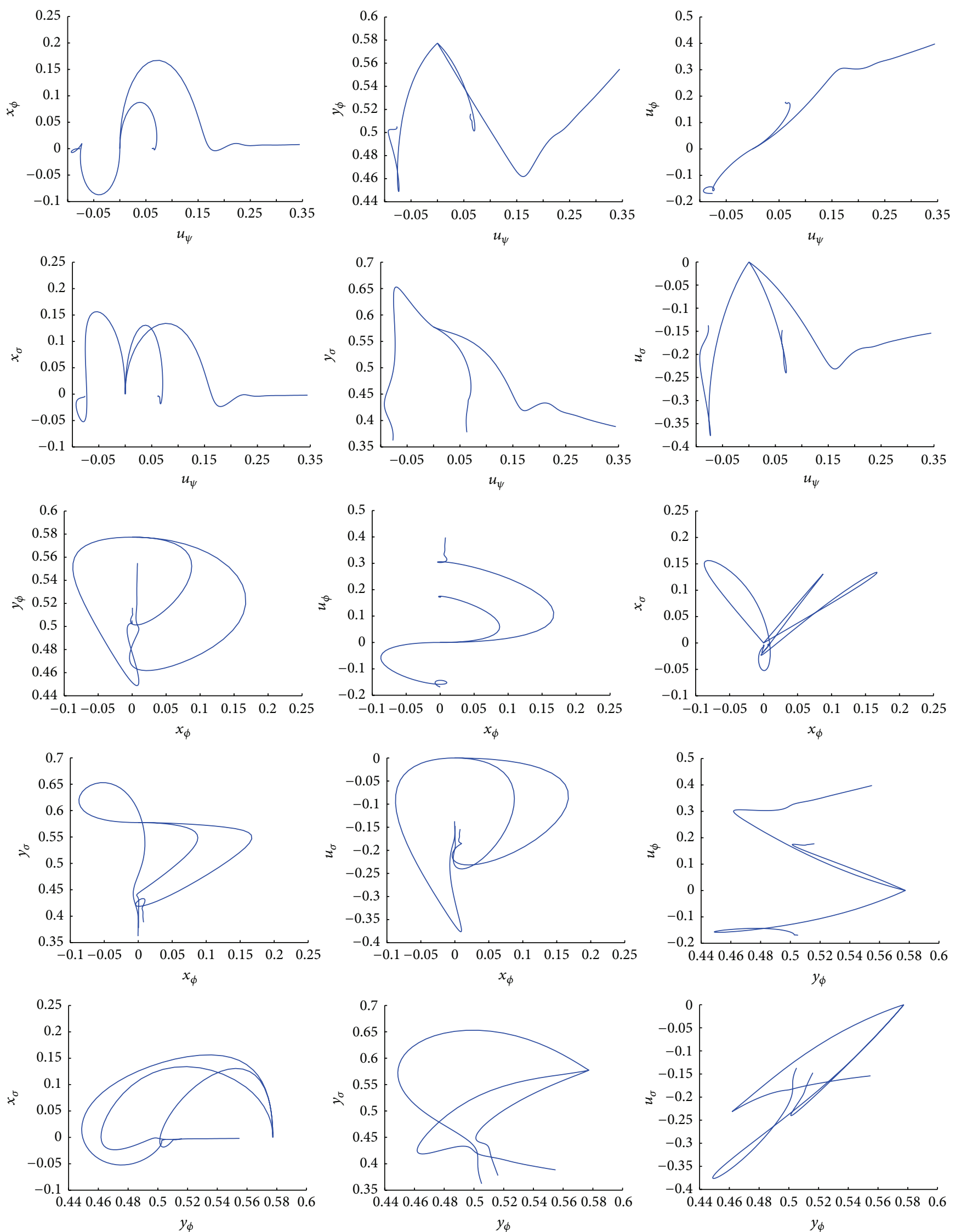

Figure 1: Continued. 

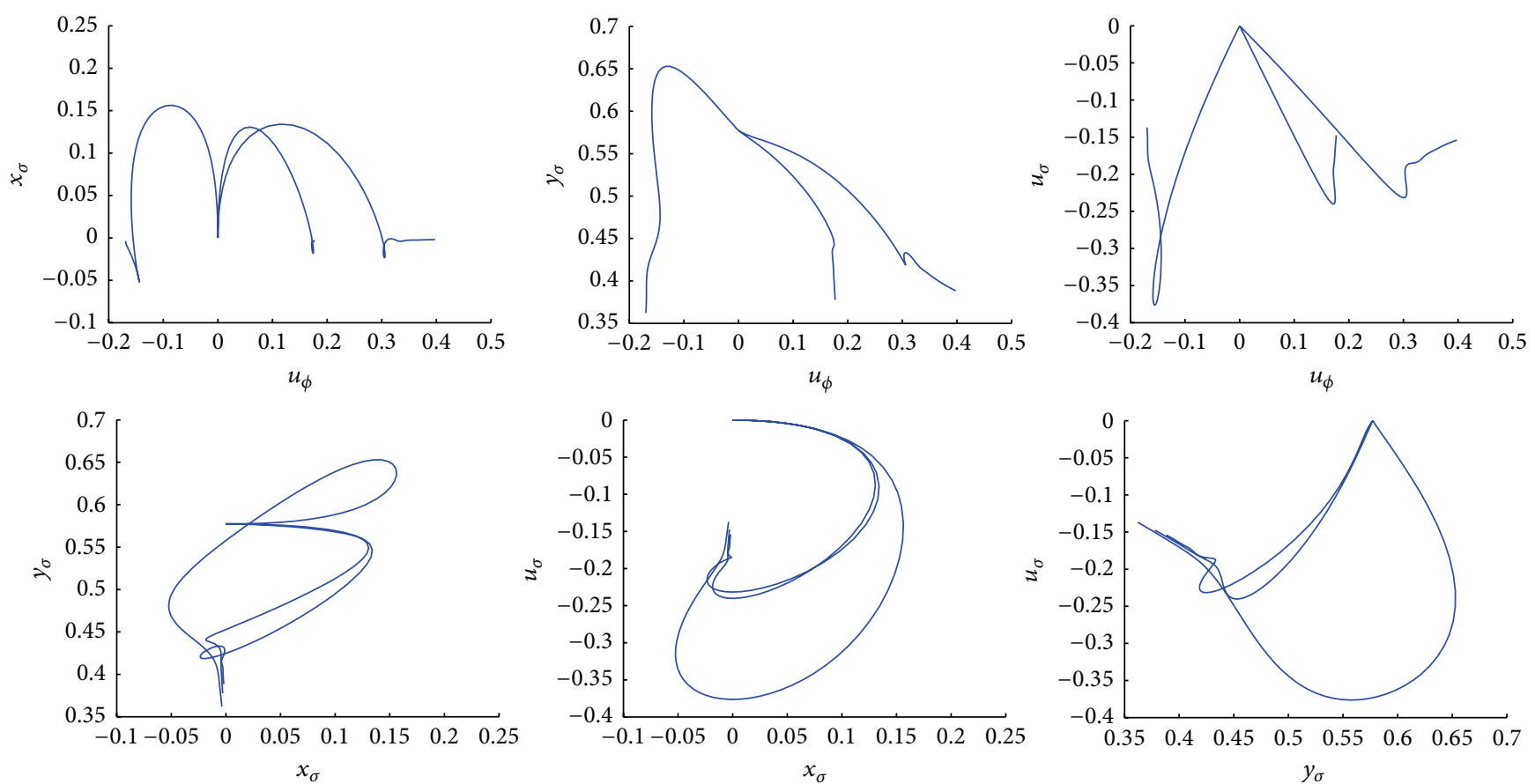

Figure 1: Two-dimensional projections of the phase-space trajectories for $\xi_{1}=\xi_{2}=-\xi_{3}=0.5, \alpha_{\psi}=\alpha_{\phi}=-\alpha_{\sigma}=1.5$. All plots begin from the critical point $A$ being a stable attractor.

TABLE 1: Critical points and existence conditions.

\begin{tabular}{|c|c|c|c|c|c|c|c|c|c|c|c|c|}
\hline Label & $x_{\psi}$ & $y_{\psi}$ & $u_{\psi}$ & $x_{\phi}$ & $y_{\phi}$ & $u_{\phi}$ & $x_{\sigma}$ & $y_{\sigma}$ & $u_{\sigma}$ & $\omega_{\text {tot }}$ & $q$ & Existence \\
\hline$A$ & 0 & $\frac{1}{\sqrt{3}}$ & 0 & 0 & $\frac{1}{\sqrt{3}}$ & 0 & 0 & $\frac{1}{\sqrt{3}}$ & 0 & -1 & -1 & $\lambda_{\psi}=\lambda_{\phi}=\lambda_{\sigma}=0$ \\
\hline$B$ & 0 & $\frac{-1}{\sqrt{3}}$ & 0 & 0 & $\frac{-1}{\sqrt{3}}$ & 0 & 0 & $\frac{-1}{\sqrt{3}}$ & 0 & -1 & -1 & $\lambda_{\psi}=\lambda_{\phi}=\lambda_{\sigma}=0$ \\
\hline C & 0 & 0 & $\frac{1}{\sqrt{6\left|\xi_{1}\right|}}$ & 0 & 0 & $\frac{1}{\sqrt{6\left|\xi_{2}\right|}}$ & 0 & 0 & $\frac{1}{\sqrt{6\left|\xi_{3}\right|}}$ & -1 & -1 & $\begin{array}{l}\alpha_{\psi}=\alpha_{\phi}=\alpha_{\sigma}=0 \\
\text { and } \\
\xi_{1}, \xi_{2}, \xi_{3}<0, \\
\lambda_{\psi}, \lambda_{\phi}, \lambda_{\sigma}<0\end{array}$ \\
\hline$D$ & 0 & 0 & $\frac{-1}{\sqrt{6\left|\xi_{1}\right|}}$ & 0 & 0 & $\frac{-1}{\sqrt{6\left|\xi_{2}\right|}}$ & 0 & 0 & $\frac{-1}{\sqrt{6\left|\xi_{3}\right|}}$ & -1 & -1 & $\begin{array}{l}\alpha_{\psi}=\alpha_{\phi}=\alpha_{\sigma}=0 \\
\text { and } \\
\xi_{1}, \xi_{2}, \xi_{3}<0, \\
\lambda_{\psi}, \lambda_{\phi}, \lambda_{\sigma}>0\end{array}$ \\
\hline
\end{tabular}

$$
\begin{aligned}
& M_{25}=-\frac{3}{P} y_{\phi} y_{\psi}, \\
& M_{26}=\frac{6 \xi_{2}}{P} u_{\phi} y_{\psi}, \\
& M_{27}=\frac{2 \sqrt{6} \alpha_{\sigma} \xi_{3}}{P} u_{\sigma} y_{\psi}, \\
& M_{28}=-\frac{3}{P} y_{\sigma} y_{\psi}, \\
& M_{29}=\frac{6 \xi_{3}}{P} u_{\sigma} y_{\psi}, \\
& M_{31}=\frac{\sqrt{3}}{2} \alpha_{\psi} y_{\psi},
\end{aligned}
$$$$
M_{44}=-3
$$$$
M_{45}=\sqrt{6} \lambda_{\phi} y_{\phi}
$$$$
M_{46}=-\sqrt{6} \alpha_{\phi} \xi_{2}
$$$$
M_{51}=2 \sqrt{6} \alpha_{\psi} \xi_{1} u_{\psi} y_{\phi}-\frac{4 \sqrt{3} \alpha_{\psi} \xi_{1}}{P}\left(\frac{P-1}{2}\right) u_{\psi} y_{\psi} y_{\phi},
$$$$
M_{52}=\frac{6}{P}\left(\frac{P-1}{2}\right) y_{\psi} y_{\phi} \text {, }
$$$$
M_{53}=-\frac{12 \xi_{1}}{P}\left(\frac{P-1}{2}\right) u_{\psi} y_{\phi} \text {, }
$$ 

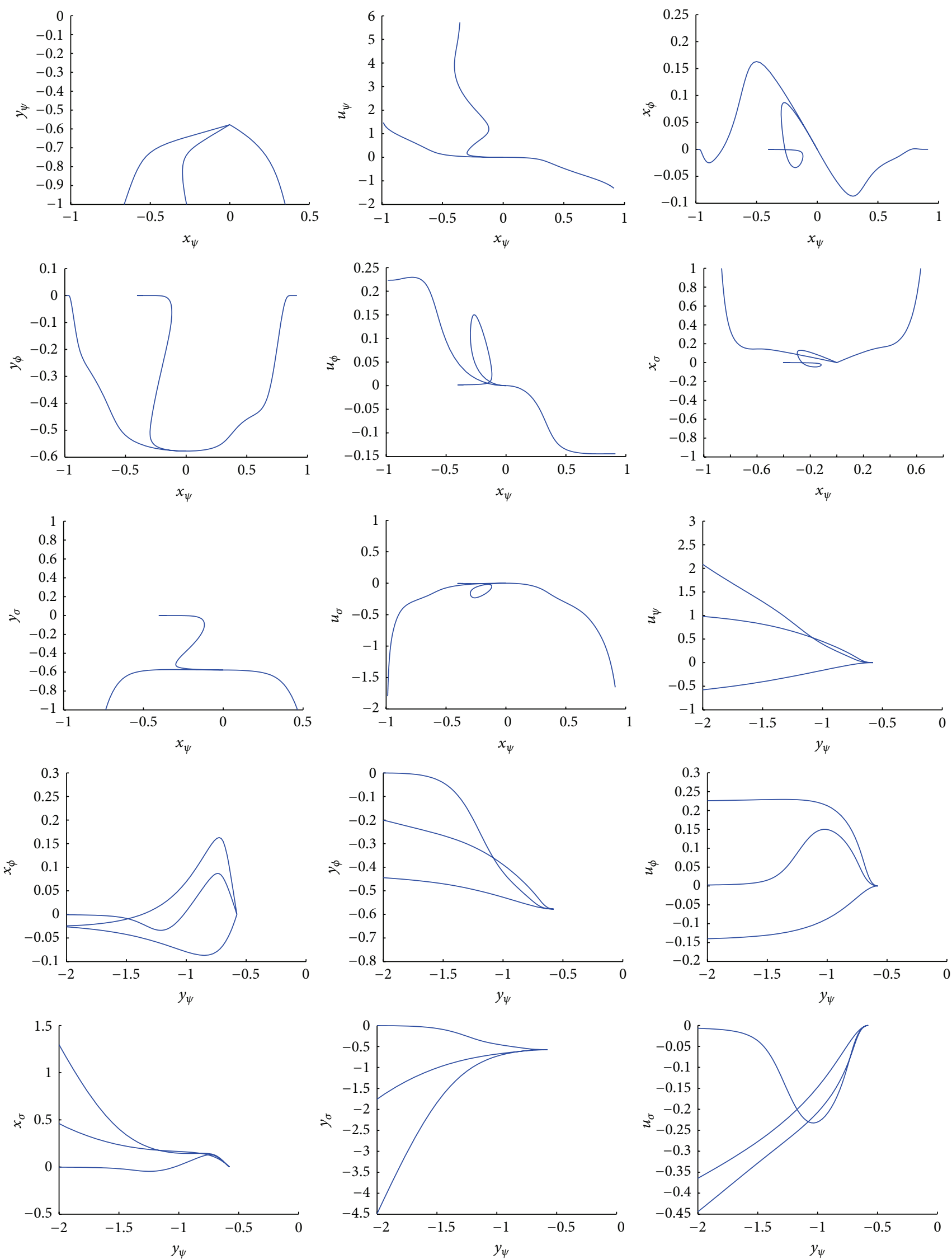

Figure 2: Continued. 

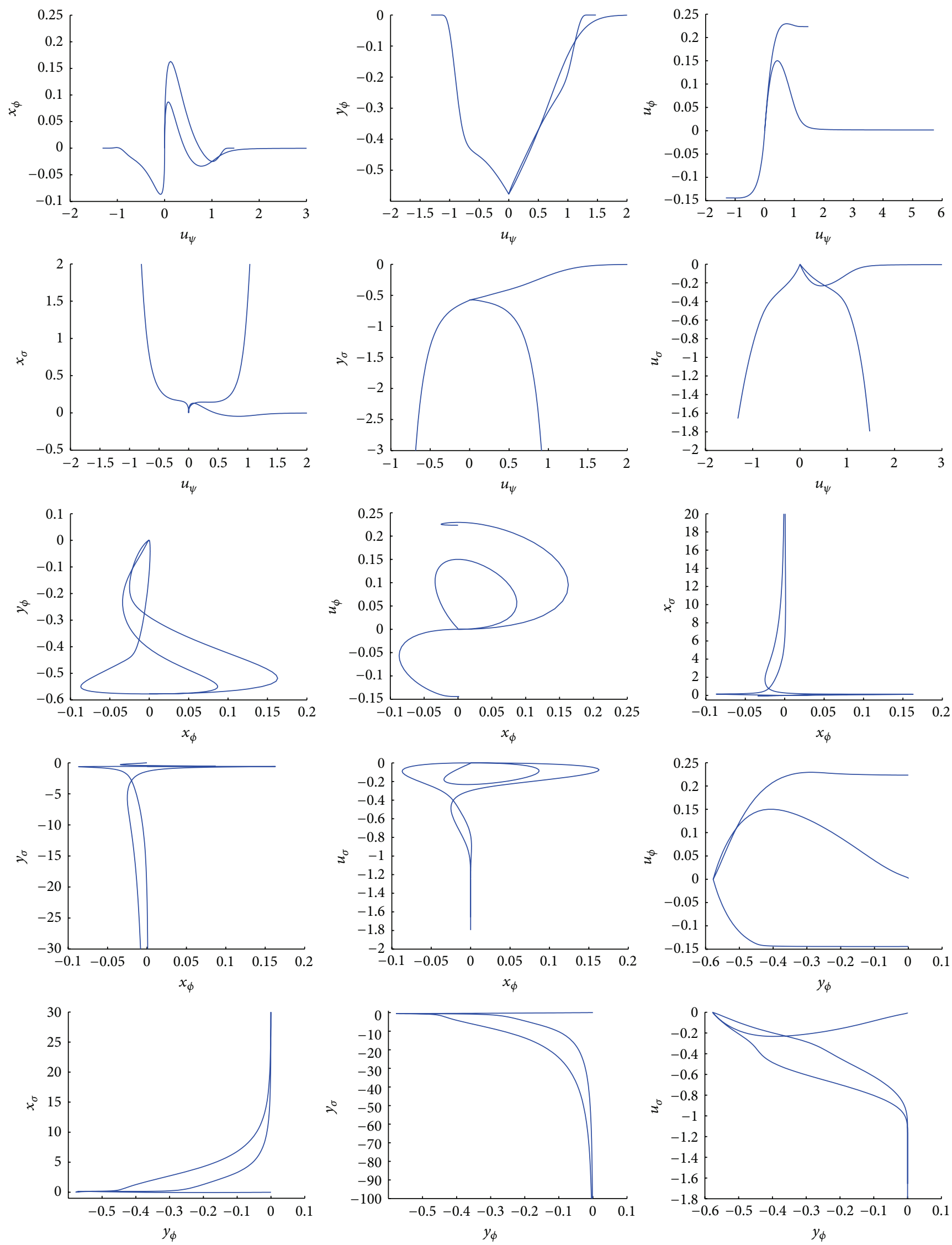

Figure 2: Continued. 

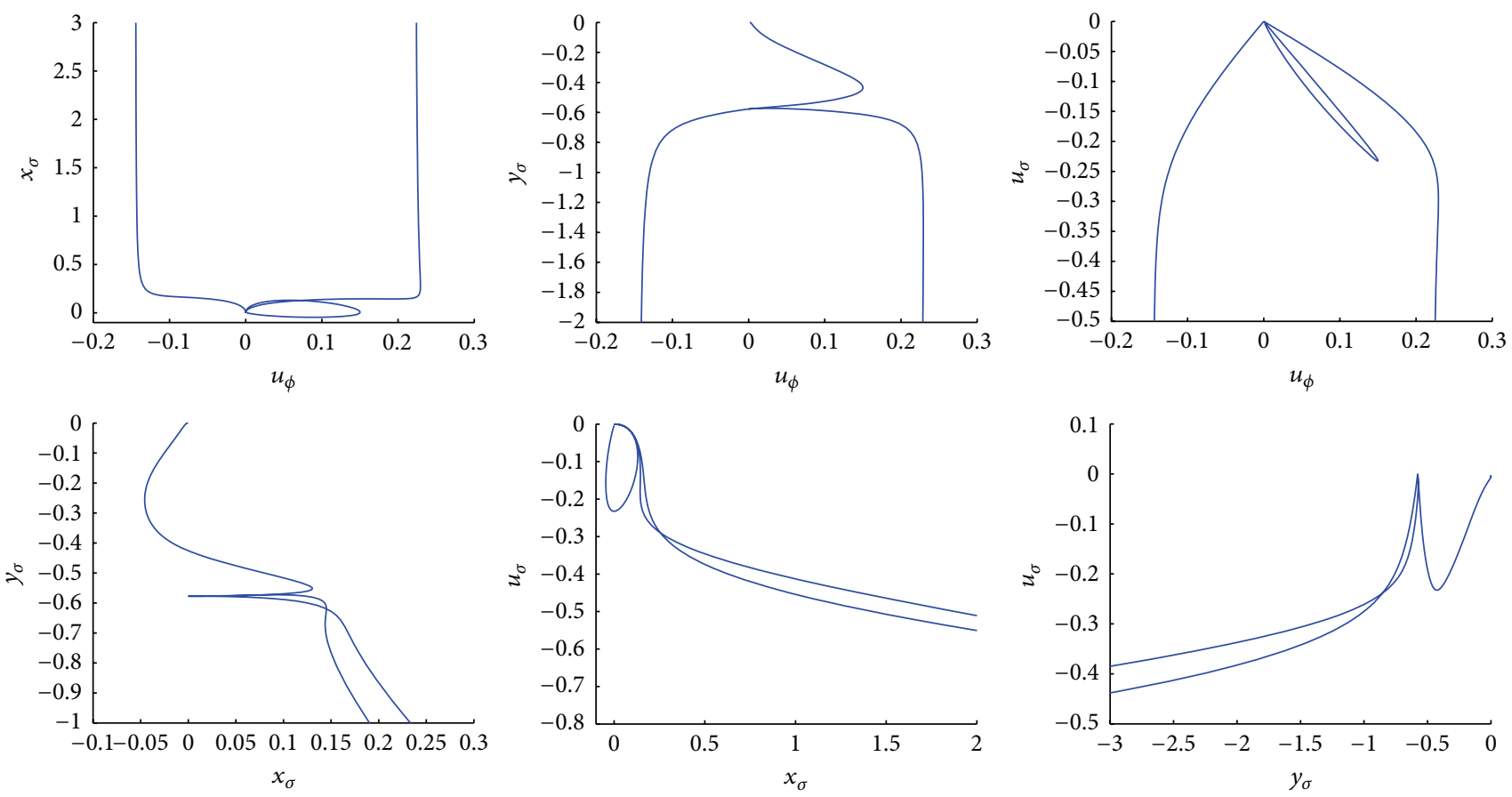

FIGURE 2: Two-dimensional projections of the phase-space trajectories for $\xi_{1}=\xi_{2}=-\xi_{3}=0.5, \alpha_{\psi}=\alpha_{\phi}=-\alpha_{\sigma}=1.5$. All plots begin from the critical point $B$ being a stable attractor.

$$
\begin{aligned}
& M_{54}=2 \sqrt{6} \alpha_{\phi} \xi_{2} u_{\phi} y_{\phi}-\frac{4 \sqrt{6} \alpha_{\phi} \xi_{2}}{P}\left(\frac{P-1}{2}\right) u_{\phi} y_{\phi} \\
& -\frac{\sqrt{6} \lambda_{\phi}}{2} y_{\phi}, \\
& M_{55}=\frac{6}{P}\left(\frac{P-1}{2}\right) y_{\phi}^{2} \text {, } \\
& M_{56}=-\frac{12 \xi_{2}}{P}\left(\frac{P-1}{2}\right) u_{\phi} y_{\phi}, \\
& M_{57}=2 \sqrt{6} \alpha_{\sigma} \xi_{3} u_{\sigma} y_{\phi}-\frac{4 \sqrt{6} \alpha_{\sigma} \xi_{3}}{P}\left(\frac{P-1}{2}\right) u_{\sigma} y_{\phi}, \\
& M_{58}=\frac{6}{P}\left(\frac{P-1}{2}\right) y_{\sigma} y_{\phi}, \\
& M_{59}=-\frac{12 \xi_{3}}{P}\left(\frac{P-1}{2}\right) u_{\sigma} y_{\phi} \text {, } \\
& M_{64}=\frac{\sqrt{6}}{2} \alpha_{\phi}, \\
& M_{77}=-3 \text {, } \\
& M_{78}=-\sqrt{6} \lambda_{\sigma} y_{\sigma}, \\
& M_{79}=\sqrt{6} \alpha_{\sigma} \xi_{3} \text {, } \\
& M_{81}=2 \sqrt{6} \alpha_{\psi} \xi_{1} u_{\psi} y_{\sigma}-\frac{4 \sqrt{3} \alpha_{\psi} \xi_{1}}{P}\left(\frac{P-1}{2}\right) u_{\psi} y_{\psi} y_{\sigma}, \\
& M_{82}=\frac{6}{P}\left(\frac{P-1}{2}\right) y_{\psi} y_{\sigma},
\end{aligned}
$$

$$
\begin{aligned}
M_{83}= & -\frac{12 \xi_{1}}{P}\left(\frac{P-1}{2}\right) u_{\psi} y_{\sigma}, \\
M_{84}= & 2 \sqrt{6} \alpha_{\phi} \xi_{2} u_{\phi} y_{\sigma}-\frac{4 \sqrt{6} \alpha_{\phi} \xi_{2}}{P}\left(\frac{P-1}{2}\right) u_{\phi} y_{\sigma}, \\
M_{85}= & \frac{6}{P}\left(\frac{P-1}{2}\right) y_{\phi} y_{\sigma}, \\
M_{86}= & -\frac{12 \xi_{2}}{P}\left(\frac{P-1}{2}\right) u_{\phi} y_{\sigma}, \\
M_{87}= & 2 \sqrt{6} \alpha_{\sigma} \xi_{3} u_{\sigma} y_{\sigma}-\frac{4 \sqrt{6} \alpha_{\sigma} \xi_{3}}{P}\left(\frac{P-1}{2}\right) u_{\sigma} y_{\sigma} \\
& -\frac{\sqrt{6} \lambda_{\sigma}}{2} y_{\sigma}, \\
M_{88}= & \frac{6}{P}\left(\frac{P-1}{2}\right) y_{\sigma}^{2}, \\
M_{89}= & -\frac{12 \xi_{3}}{P}\left(\frac{P-1}{2}\right) u_{\sigma} y_{\sigma}, \\
M_{97}= & \frac{\sqrt{6}}{2} \alpha_{\sigma},
\end{aligned}
$$

and all the other matrix elements except those given above in (27) are zero. Here also $P=1+2 \xi_{1} u_{\psi}^{2}+2 \xi_{2} u_{\phi}^{2}+$ $2 \xi_{3} u_{\sigma}^{2}$. Then we calculate the eigenvalues of perturbation matrix $M$ for four critical points given in Table 1 with the corresponding existing conditions. We obtain four sets of eigenvalues for four perturbation matrices for each of the four critical points. To determine the type and stability of critical points, we examine the sign of the real parts of eigenvalues. 

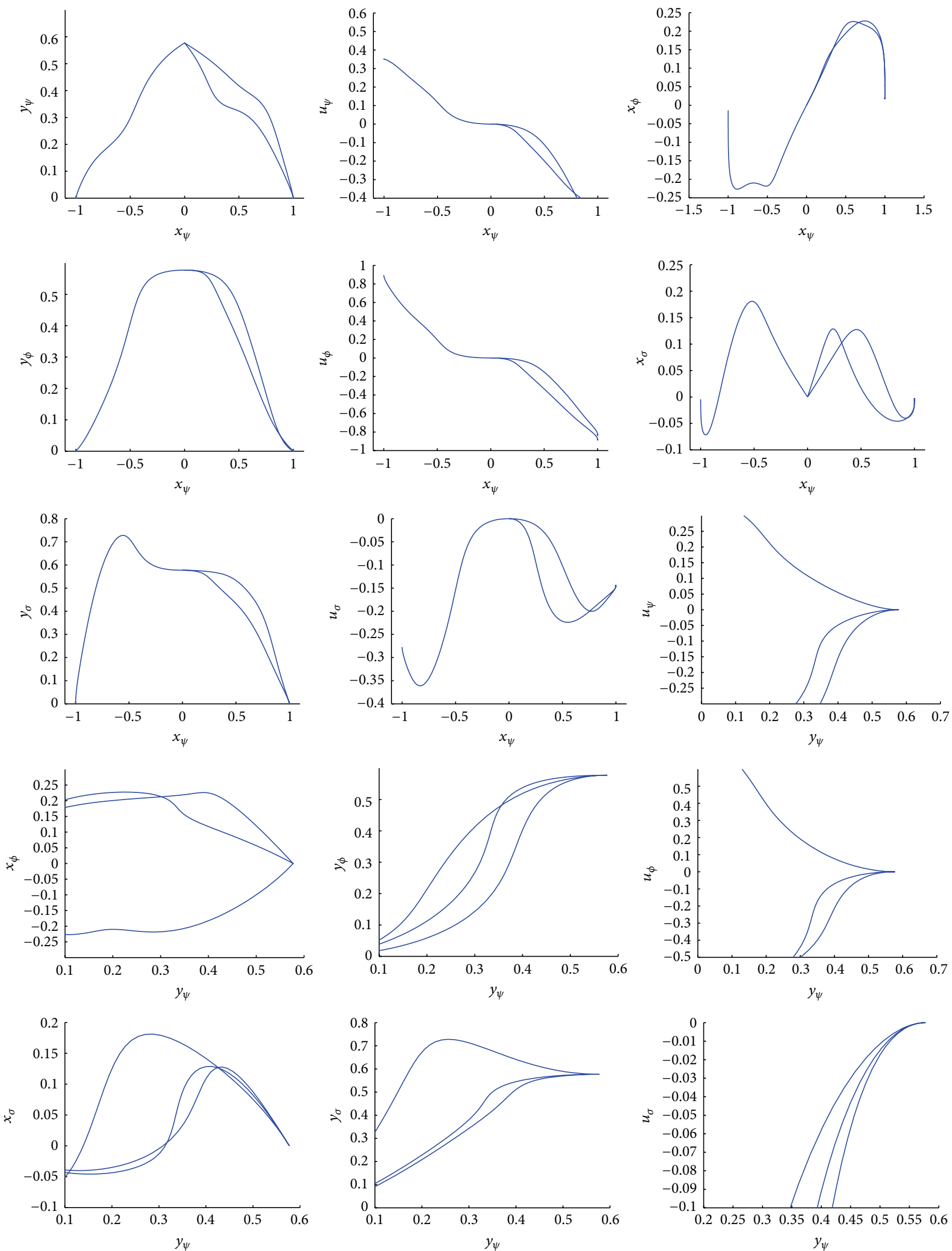

FIgURE 3: Continued. 

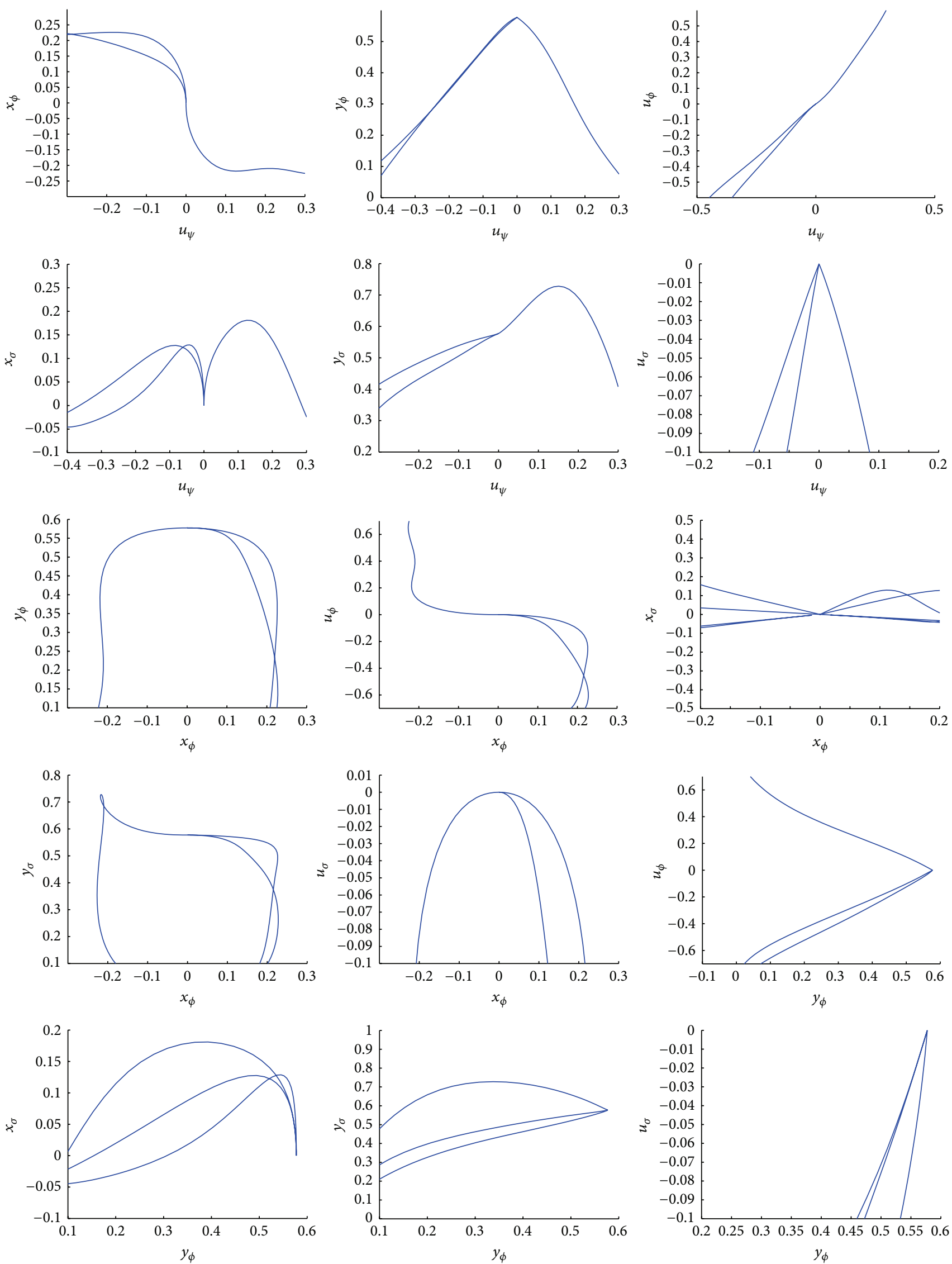

Figure 3: Continued. 

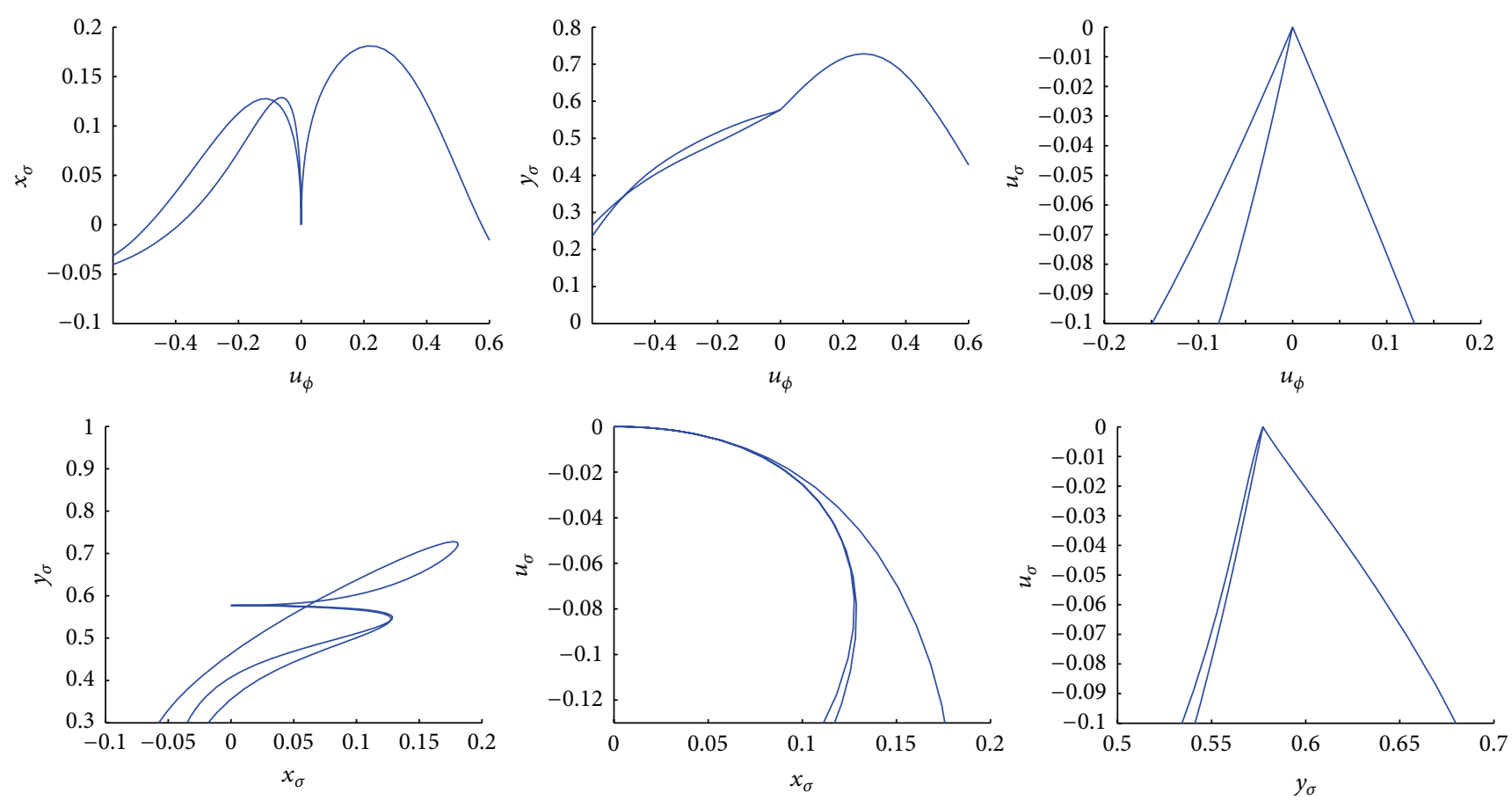

Figure 3: Two-dimensional projections of the phase-space trajectories for $\xi_{1}=\xi_{2}=\xi_{3}=-0.5, \alpha_{\psi}=\alpha_{\phi}=\alpha_{\sigma}=-1.5$. All plots begin from the critical point $C$ being a stable attractor.

TABLE 2: Eigenvalues of the perturbation matrix for each critical point.

\begin{tabular}{ll}
\hline Critical points & Eigenvalues \\
\hline & $\frac{1}{2} \sqrt{9-12 \xi_{1} \alpha_{\psi}^{2}}-\frac{3}{2}$, \\
& $-\frac{1}{2} \sqrt{9-12 \xi_{1} \alpha_{\psi}^{2}}-\frac{3}{2}$, \\
& $\frac{1}{2} \sqrt{9-12 \xi_{2} \alpha_{\phi}^{2}}-\frac{3}{2}$, \\
$A$ and $B$ & $-\frac{1}{2} \sqrt{9-12 \xi_{2} \alpha_{\phi}^{2}}-\frac{3}{2}$, \\
& $\frac{1}{2} \sqrt{12 \xi_{3} \alpha_{\sigma}^{2}+9}-\frac{3}{2}$, \\
& $-\frac{1}{2} \sqrt{12 \xi_{3} \alpha_{\sigma}^{2}+9}-\frac{3}{2}$, \\
\hline$C$ and $D$ & -1 \\
\hline
\end{tabular}

The critical point is stable for negative real part of eigenvalues. The physical meaning of negative eigenvalue is always a latetime stable attractor; namely, if the universe reaches this solution, it keeps its state forever and thus it can attract the universe at a late-time. Accelerated expansion occurs here, because $\omega_{\text {tot }}=-1<-1 / 3$. If a convenient condition is provided, an accelerated contraction can even exist for $\omega_{\text {tot }}=$ $-1<-1 / 3$ value. Eigenvalues of the matrix $M$ are represented in Table 2 for each of the critical points $A, B, C$, and $D$.

As seen in Table 2, the first two critical points $A$ and $B$ have the same eigenvalues as $C$ and $D$ have same eigenvalues. The stability conditions of each critical point are listed in Table 3, according to the sign of the eigenvalues.
TABLE 3: Stability of the critical points.

\begin{tabular}{ll}
\hline Critical points & Stability \\
\hline & Stable point, if $0<\frac{3}{4 \alpha_{\psi}^{2}}<\xi_{1}$, \\
& $0<\frac{3}{4 \alpha_{\phi}^{2}}<\xi_{2}$, \\
& and $\xi_{3}<\frac{-3}{4 \alpha_{\sigma}^{2}}<0$ \\
& Saddle point, if $\xi_{1}, \xi_{2}<0$ \\
\hline$C$ and $D$ & Stable point for all $\xi, \alpha$, and $\lambda$ \\
\hline
\end{tabular}

In order to analyze the cosmological behavior of each critical point, the attractor solutions in scalar field cosmology should be noted [64]. In modern theoretical cosmology it is common that the energy density of one or more scalar fields exerts a crucial influence on the evolution of the universe and some certain conditions or behaviors naturally affect this evolution. The evolution and the affecting factors on this evolution meet in the term of cosmological attractors: the scalar field evolution approaches a certain kind of behavior by the dynamical conditions without finely tuned initial conditions [65-77], either in inflationary cosmology or in late-time dark energy models. Attractor behavior is a situation in which a collection of phase-space points evolve into a certain region and never leave.

Critical Point A. This point exists for all values of $\xi_{1}, \xi_{2}, \xi_{3}$, $\alpha_{\psi}, \alpha_{\phi}$, and $\alpha_{\sigma}$ while $\lambda_{\psi}=\lambda_{\phi}=\lambda_{\sigma}=0$ which means the potentials $V_{\psi}, V_{\phi}$, and $V_{\sigma}$ are constant. Acceleration occurs at this point since $\omega_{\text {tot }}=-1<-1 / 3$ and this is an expansion 

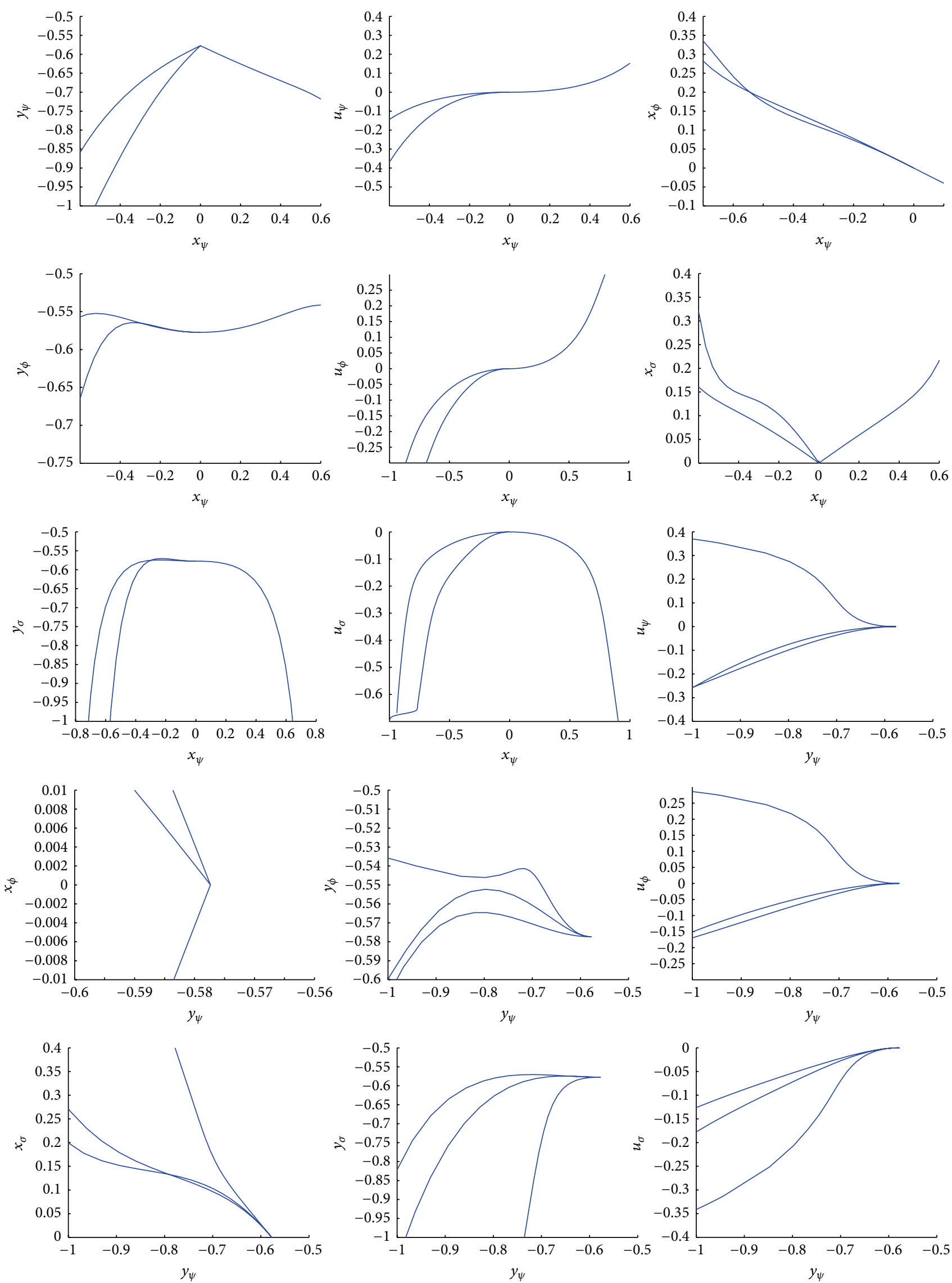

Figure 4: Continued. 

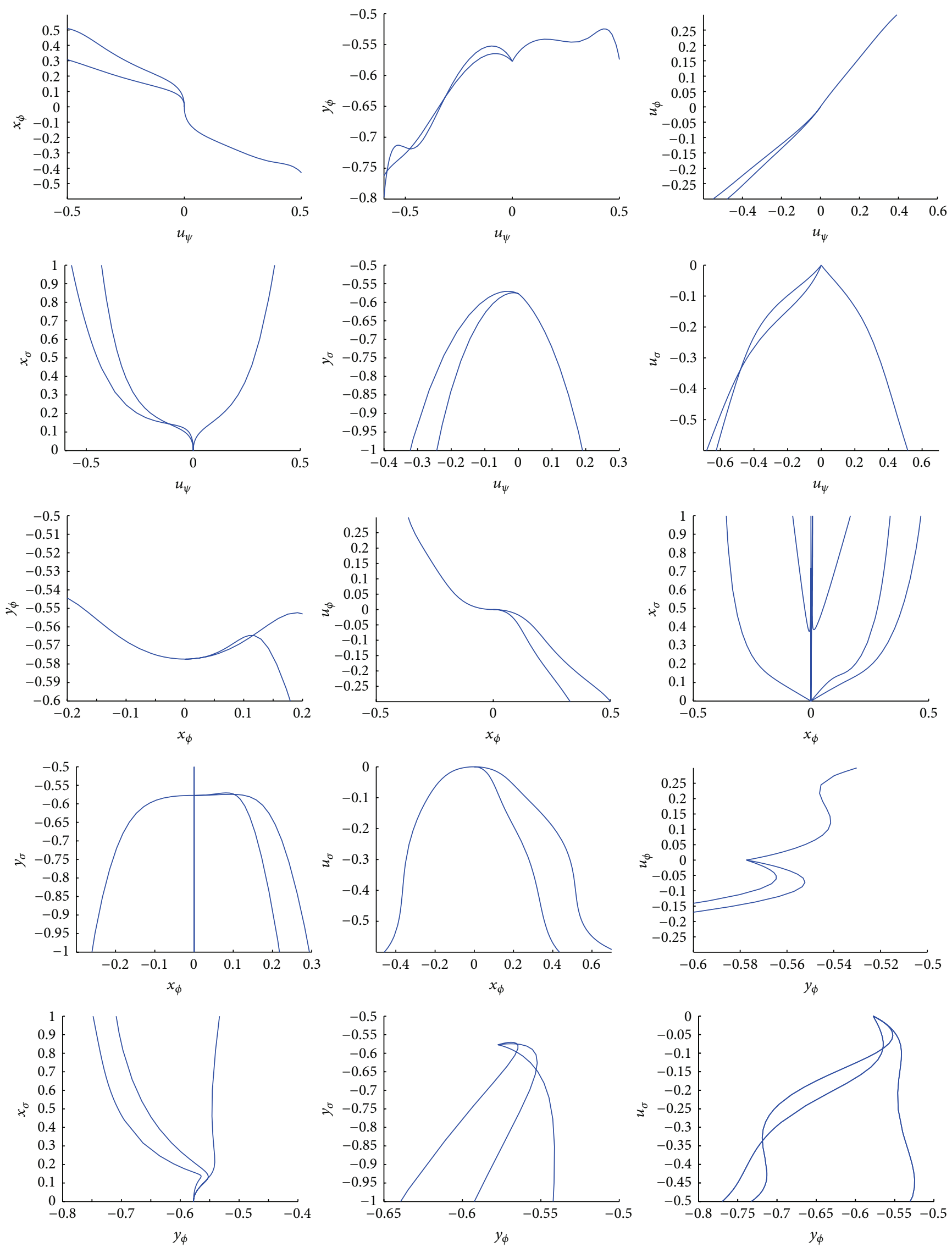

Figure 4: Continued. 

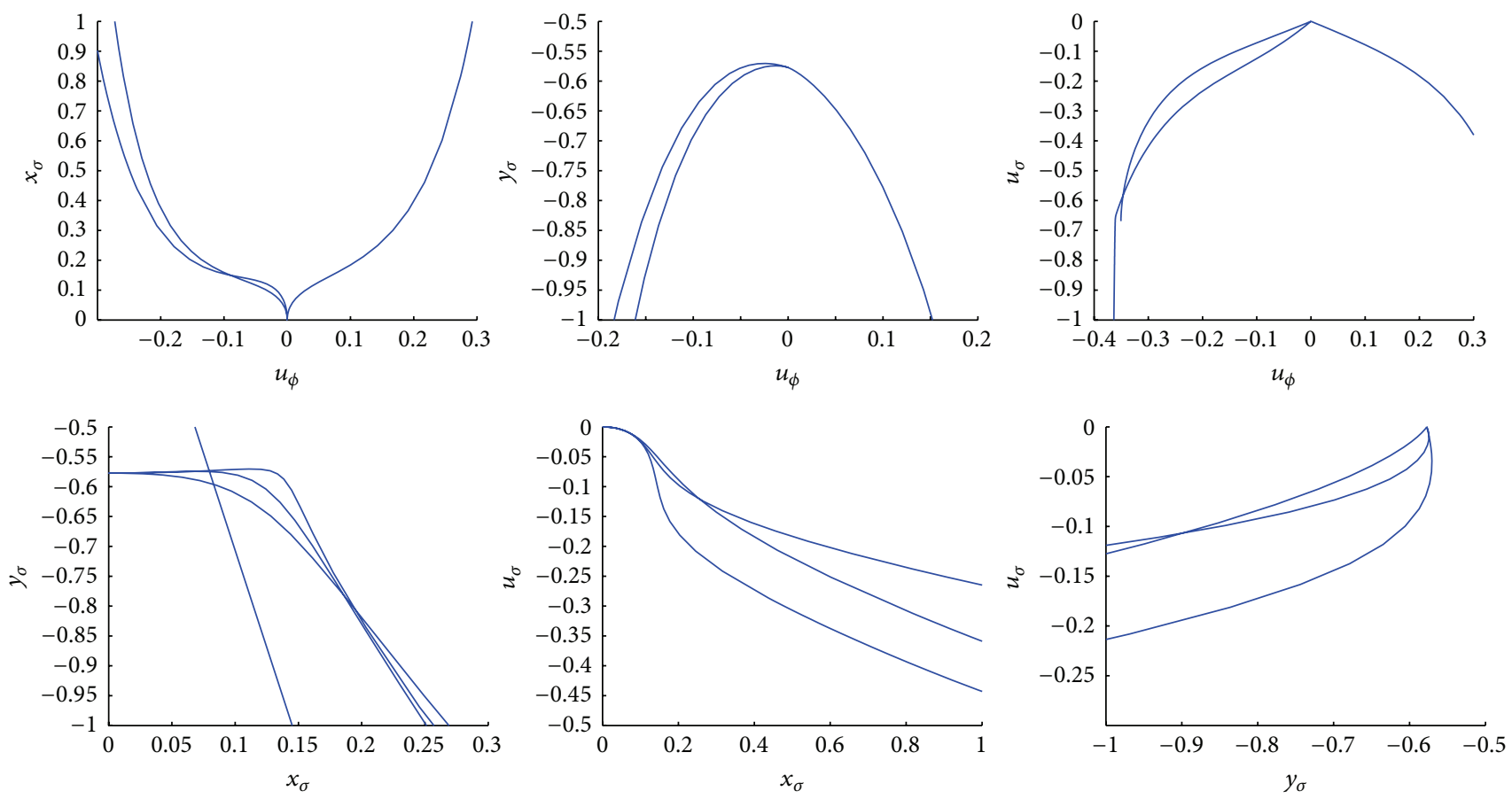

Figure 4: Two-dimensional projections of the phase-space trajectories for $\xi_{1}=\xi_{2}=\xi_{3}=-0.5, \alpha_{\psi}=\alpha_{\phi}=\alpha_{\sigma}=-1.5$. All plots begin from the critical point $D$ being a stable attractor.

phase because $y_{\psi}, y_{\phi}$, and $y_{\sigma}$ have positive values; therefore $H$ is positive, too. Point $A$ is stable (meaning that universe keeps its evolution), if $0<3 /\left(4 \alpha_{\psi}^{2}\right)<\xi_{1}, 0<3 /\left(4 \alpha_{\phi}^{2}\right)<\xi_{2}$ and $\xi_{3}<-3 /\left(4 \alpha_{\sigma}^{2}\right)<0$, but it is a saddle point (meaning that the universe evolves between different states) for values $\xi_{1}, \xi_{2}<0$. In Figure 1, we represent the 2-dimensional projections of 9dimensional phase-space trajectories for the values of $\xi_{1}=$ $\xi_{2}=-\xi_{3}=0.5, \alpha_{\psi}=\alpha_{\phi}=-\alpha_{\sigma}=1.5$ and three auxiliary $\lambda$ values for each field $\psi, \phi$, and $\sigma$. This state corresponds to a stable attractor starting from the critical point $A$, as seen from the plots in Figure 1.

Critical Point $B$. Point $B$ also exists for any values of $\xi_{1}, \xi_{2}, \xi_{3}$, $\alpha_{\psi}, \alpha_{\phi}$, and $\alpha_{\sigma}$ while $\lambda_{\psi}=\lambda_{\phi}=\lambda_{\sigma}=0$ which means the potentials $V_{\psi}, V_{\phi}$, and $V_{\sigma}$ are constant. Acceleration phase is again valid here since $\omega_{\text {tot }}=-1<-1 / 3$, but this point refers to contraction phase because $y_{\psi}, y_{\phi}$, and $y_{\sigma}$ have negative sign. Stability of the point $B$ is same with the point $A$ for same conditions. Therefore the stable attractor behavior for contraction is represented in Figure 2. We plot 2-dimensional projections of phase-space trajectories for same values as point $A, \xi_{1}=\xi_{2}=-\xi_{3}=0.5, \alpha_{\psi}=\alpha_{\phi}=-\alpha_{\sigma}=1.5$, and auxiliary $\lambda$ values.

Critical Point C. Critical point $C$ occurs for $\xi_{1}, \xi_{2}, \xi_{3}<0$ and $\lambda_{\psi}, \lambda_{\phi}, \lambda_{\sigma}<0$ while $\alpha_{\psi}=\alpha_{\phi}=\alpha_{\sigma}=0$ meaning constant coupling functions $f(\psi), g(\phi)$, and $h(\sigma)$. The cosmological behavior is again an acceleration phase since $\omega_{\text {tot }}<-1 / 3$. Point $C$ is stable for any values of $\xi, \alpha$, and $\lambda$ of fields $\psi$, $\phi$, and $\sigma$. Two-dimensional projections of phase-space are represented in Figure 3 for $\xi_{1}=\xi_{2}=\xi_{3}=-0.5, \alpha_{\psi}=\alpha_{\phi}=$ $\alpha_{\sigma}=-1.5$, and three auxiliary $\lambda$ values for each field $\psi, \phi$, and $\sigma$. A stable attractor starting from the critical point $C$ is seen from the plots in Figure 3.

Critical Point D. This point exists for $\xi_{1}, \xi_{2}, \xi_{3}<0$ and $\lambda_{\psi}, \lambda_{\phi}, \lambda_{\sigma}>0$ while $\alpha_{\psi}=\alpha_{\phi}=\alpha_{\sigma}=0$ implying constant $f(\psi), g(\phi), h(\sigma)$ coupling functions. Acceleration phase is valid due to $\omega_{\text {tot }}<-1 / 3$. Point $D$ is also stable for all $\xi, \alpha$, and $\lambda$ values of fields $\psi, \phi$, and $\sigma$. Two-dimensional plots of phase-space trajectories are shown in Figure 4 for $\xi_{1}=\xi_{2}=$ $\xi_{3}=-0.5, \alpha_{\psi}=\alpha_{\phi}=\alpha_{\sigma}=-1.5$, and three auxiliary $\lambda$ values for each field $\psi, \phi$, and $\sigma$. This state again corresponds to a stable attractor starting from the point $C$, as in Figure 4 .

All the plots in Figures 1-4 have the structure of stable attractor, since each of them evolves to a single point which is in fact one of the critical points in Table 1. These evolutions to the critical points are the attractor solutions in mixed dark energy domination cosmology of our model which imply an expanding universe.

\section{Conclusion}

Mixed dark energy is a generalized combination of tachyon, quintessence, and phantom fields nonminimally coupled to gravity $[33,34,38,39]$. These three scalar fields are the constituents of mixed dark energy. Firstly, the action integral of nonminimally coupled mixed dark energy model is set up to study its dynamics. Here we consider that our dark energy constituents interact only with the gravity. There could also be chosen some interactions between the dark constituents, but in order to start from a pedagogical order we 
prefer to exclude the dark interactions. We obtain the Hubble parameter and Friedmann equations of model in spatially flat FRW geometry. Energy density and pressure values with the evolution equations for tachyon, quintessence, and phantom fields are obtained from the variation of action and Lagrangian of model. Then we translate these dynamical expressions into the autonomous form by introducing suitable auxiliary variables in order to perform the phasespace analysis. We find the critical points of autonomous system by setting each autonomous equation to zero. By constructing the perturbation equations, we find four perturbation matrices for each critical point. The eigenvalues of four perturbation matrices are determined to examine the stability of critical points. We also calculate some important cosmological parameters, for instance, the total equation of state parameter and deceleration parameter to see whether the critical points correspond to an accelerating universe or not. There are four stable attractors of model depending on the nonminimal coupling parameters $\xi_{1}, \xi_{2}, \xi_{3}$ and $\alpha_{\psi}$, $\alpha_{\phi}, \alpha_{\sigma}$ values. All of the stable solutions correspond to an accelerating universe due to $\omega_{\text {tot }}<-1 / 3$. For constant potentials $V_{\psi}, V_{\phi}$, and $V_{\sigma}$ the critical points $A$ and $B$ are latetime stable attractors for $0<3 /\left(4 \alpha_{\psi}^{2}\right)<\xi_{1}, 0<3 /\left(4 \alpha_{\phi}^{2}\right)<\xi_{2}$, and $\xi_{3}<-3 /\left(4 \alpha_{\sigma}^{2}\right)<0$. While point $A$ refers to an expansion with a stable acceleration, point $B$ refers to a contraction. However, for constant coupling functions $f(\psi), g(\phi)$, and $h(\sigma)$ the critical points $C$ and $D$ are stable attractors for any values of $\xi_{1}, \xi_{2}, \xi_{3}, \alpha_{\psi}, \alpha_{\phi}$, and $\alpha_{\sigma}$. The behavior of the model at each critical point being a stable attractor is demonstrated in Figures 1-4. In order to plot the graphs in Figures 1-4, we use adaptive Runge-Kutta method of 4 th and 5th order to solve differential equations (26) in Matlab. Solutions for the equations with stability conditions of critical points are plotted for each pair of the solution being the auxiliary variables in (19).

These figures show that, by choosing parameters of the model depending on the existence conditions of critical points $A, B, C$, and $D$, we obtain the attractors of the model as $A, B, C$, and $D$. Then depending on the stability conditions, suitable parameters give stable behavior for each attractor of the model. The results are consistent with the observed and expected behavior of the universe in which some epochs correspond to an accelerating expansion phase, and some will correspond to an accelerating contraction in future times [1$9,76,77]$.

\section{Conflict of Interests}

The authors declare that there is no conflict of interests regarding the publication of this paper.

\section{References}

[1] S. Perlmutter, G. Aldering, G. Goldhaber et al., "Measurements of $\Omega$ and $\Lambda$ from 42 high-redshift supernovae," The Astrophysical Journal, vol. 517, no. 2, pp. 565-586, 1999.

[2] A. G. Riess, A. V. Filippenko, P. Challis et al., "Observational evidence from supernovae for an accelerating universe and a cosmological constant," The Astronomical Journal, vol. 116, no. 3, pp. 1009-1038, 1998.

[3] U. Seljak, A. Makarov, P. McDonald et al., "Cosmological parameter analysis including SDSS Ly $\alpha$ forest and galaxy bias: constraints on the primordial spectrum of fluctuations, neutrino mass, and dark energy," Physical Review D, vol. 71, no. 10, Article ID 103515, 20 pages, 2005.

[4] M. Tegmark, M. Strauss, M. Blanton et al., "Cosmological parameters from SDSS and WMAP," Physical Review D, vol. 69, Article ID 103501, 2004.

[5] D. J. Eisenstein, I. Zehavi, D. W. Hogg et al., "Detection of the baryon acoustic peak in the large-scale correlation function of SDSS luminous red galaxies," The Astrophysical Journal, vol. 633, no. 2, pp. 560-574, 2005.

[6] D. N. Spergel, L. Verde, H. V. Peiris et al., "First-year Wilkinson Microwave Anisotropy Probe (WMAP) observations: determination of cosmological parameters," The Astrophysical Journal Supplement Series, vol. 148, no. 1, pp. 175-194, 2003.

[7] E. Komatsu, K. M. Smith, J. Dunkley et al., "Seven-year Wilkinson microwave anisotropy probe (WMAP) observations: cosmological interpretation," The Astrophysical Journal Supplement Series, vol. 192, no. 2, article 18, 2011.

[8] G. Hinshaw, D. Larson, E. Komatsu et al., "Nine-year Wilkinson microwave anisotropy probe (WMAP) observations: cosmological parameter results," The Astrophysical Journal Supplement Series, vol. 208, no. 2, p. 19, 2013.

[9] P. A. R. Ade, N. Aghanim, C. Armitage-Caplan et al., "Planck 2013 results. XVI. Cosmological parameters,” Astronomy \& Astrophysics, vol. 571, article A16, 2013.

[10] S. Weinberg, "The cosmological constant problem," Reviews of Modern Physics, vol. 61, no. 1, pp. 1-23, 1989.

[11] S. M. Carroll, W. H. Press, and E. L. Turner, "The cosmological constant," Annual Review of Astronomy and Astrophysics, vol. 30, no. 1, pp. 499-542, 1992.

[12] L. M. Krauss and M. S. Turner, "The cosmological constant is back," General Relativity and Gravitation, vol. 27, no. 11, pp. 1137$1144,1995$.

[13] G. Huey, L. Wang, R. Dave, R. R. Caldwell, and P. J. Steinhardt, "Resolving the cosmological missing energy problem," Physical Review D, vol. 59, Article ID 063005, 1999.

[14] S. M. Carroll, “The cosmological constant," Living Reviews in Relativity, vol. 4, no. 1, 2001.

[15] R.-J. Yang and S. N. Zhang, "The age problem in the $\Lambda$ CDM model," Monthly Notices of the Royal Astronomical Society, vol. 407, no. 3, pp. 1835-1841, 2010.

[16] Y.-F. Cai, E. N. Saridakis, M. R. Setare, and J.-Q. Xia, "Quintom cosmology: theoretical implications and observations," Physics Reports, vol. 493, no. 1, pp. 1-60, 2010.

[17] K. Bamba, S. Capozziello, S. Nojiri, and S. D. Odintsov, "Dark energy cosmology: the equivalent description via different theoretical models and cosmography tests," Astrophysics and Space Science, vol. 342, no. 1, pp. 155-228, 2012.

[18] A. Banijamali and B. Fazlpour, "Tachyonic teleparallel dark energy," Astrophysics and Space Science, vol. 342, no. 1, pp. 229$235,2012$.

[19] J.-Q. Xia, Y.-F. Cai, T.-T. Qiu, G.-B. Zhao, and X. Zhang, "Constraints on the sound speed of dynamical dark energy," International Journal of Modern Physics D, vol. 17, no. 8, pp. 1229-1243, 2008.

[20] A. Vikman, "Can dark energy evolve to the phantom?" Physical Review D, vol. 71, no. 2, Article ID 023515, 14 pages, 2005. 
[21] W. Hu, "Crossing the phantom divide: dark energy internal degrees of freedom," Physical Review D, vol. 71, Article ID 047301, 2005.

[22] R. R. Caldwell and M. Doran, "Dark-energy evolution across the cosmological-constant boundary," Physical Review D, vol. 72, no. 4, Article ID 043527, 6 pages, 2005.

[23] G.-B. Zhao, J.-Q. Xia, M. Li, B. Feng, and X. Zhang, "Perturbations of the quintom models of dark energy and the effects on observations," Physical Review D, vol. 72, no. 12, Article ID 123515, 2005.

[24] M. Kunz and D. Sapone, "Crossing the phantom divide," Physical Review D, vol. 74, no. 12, Article ID 123503, 2006.

[25] L. Amendola, “Coupled quintessence," Physical Review D, vol. 62, no. 4, Article ID 043511, 10 pages, 2000.

[26] B. Gumjudpai, T. Naskar, M. Sami, and S. Tsujikawa, "Coupled dark energy: towards a general description of the dynamics," Journal of Cosmology and Astroparticle Physics, vol. 2005, no. 6, article 007, 2005.

[27] A. Einstein, "Riemanngeometrie mit Aufrechterhaltung des Begriffes des Fern-Parallelismus," in Sitzungsberichte der Preussischen Akademie der Wissenschaften, pp. 217-221, Physikalisch-Mathematische Klasse, 1928.

[28] K. Hayashi and T. Shirafuji, "New general relativity," Physical Review D, vol. 19, no. 12, pp. 3524-3553, 1979.

[29] K. Hayashi and T. Shirafuji, "Addendum to 'new general relativity"' Physical Review D, vol. 24, no. 12, p. 3312, 1981.

[30] R. Ferraro and F. Fiorini, "Modified teleparallel gravity: inflation without an inflaton," Physical Review D, vol. 75, Article ID 084031, 2007.

[31] G. R. Bengochea and R. Ferraro, "Dark torsion as the cosmic speed-up,” Physical Review D, vol. 79, no. 12, Article ID 124019, 2009.

[32] E. V. Linder, "Einstein's other gravity and the acceleration of the Universe," Physical Review D, vol. 81, no. 12, Article ID 127301, 2010.

[33] C.-Q. Geng, C.-C. Lee, E. N. Saridakis, and Y.-P. Wu, “'Teleparallel' dark energy," Physics Letters B, vol. 704, no. 5, pp. 384-387, 2011.

[34] C.-Q. Geng, C.-C. Lee, and E. N. Saridakis, "Observational constraints on teleparallel dark energy," Journal of Cosmology and Astroparticle Physics, vol. 2012, no. 1, article 002, 2012.

[35] C. Xu, E. N. Saridakis, and G. Leon, "Phase-space analysis of teleparallel dark energy," Journal of Cosmology and Astroparticle Physics, vol. 2012, no. 7, article 005, 2012.

[36] H. Wei, "Dynamics of teleparallel dark energy," Physics Letters $B$, vol. 712, no. $4-5$, pp. $430-436,2012$.

[37] G. Otalora, "Scaling attractors in interacting teleparallel dark energy," Journal of Cosmology and Astroparticle Physics, vol. 2013, no. 7, article 44, 2013.

[38] A. Banijamali, "Dynamics of interacting tachyonic teleparallel dark energy," Advances in High Energy Physics, vol. 2014, Article ID 631630, 14 pages, 2014.

[39] B. Fazlpour and A. Banijamali, "Tachyonic teleparallel dark energy in phase space," Advances in High Energy Physics, vol. 2013, Article ID 279768, 9 pages, 2013.

[40] G. Otalora, "Cosmological dynamics of tachyonic teleparallel dark energy," Physical Review D, vol. 88, no. 6, Article ID 063505, 8 pages, 2013.

[41] R. Weitzenböck, Invarianten Theorie, Nordho, Groningen, The Netherlands, 1923.
[42] K. Hayashi and T. Shirafuji, "New general relativity," Physical Review D, vol. 19, article 3524, 1979.

[43] E. J. Copeland, M. Sami, and S. Tsujikawa, "Dynamics of dark energy," International Journal of Modern Physics. D, vol. 15, no. 11, pp. 1753-1935, 2006.

[44] P. G. Ferreira and M. Joyce, "Structure formation with a selftuning scalar field," Physical Review Letters, vol. 79, no. 24, pp. 4740-4743, 1997.

[45] E. J. Copeland, A. R. Liddle, and D. Wands, "Exponential potentials and cosmological scaling solutions," Physical Review $D$, vol. 57, no. 8, pp. 4686-4690, 1998.

[46] X. M. Chen, Y. G. Gong, and E. N. Saridakis, "Phase-space analysis of interacting phantom cosmology," Journal of Cosmology and Astroparticle Physics, vol. 2009, no. 4, article 001, 2009.

[47] A. R. Liddle, P. Parsons, and J. D. Barrow, "Formalizing the slowroll approximation in inflation," Physical Review D, vol. 50, no. 12, pp. 7222-7232, 1994.

[48] P. G. Ferreira and M. Joyce, "Cosmology with a primordial scaling field," Physical Review D, vol. 58, no. 2, Article ID 023503, 1998.

[49] Y. Gong, A. Wang, and Y.-Z. Zhang, "Exact scaling solutions and fixed points for general scalar field," Physics Letters B, vol. 636, no. 5, pp. 286-292, 2006.

[50] X.-M. Chen and Y. Gong, "Fixed points in interacting dark energy models," Physics Letters B, vol. 675, no. 1, pp. 9-13, 2009.

[51] Z.-K. Guo, Y.-S. Piao, X. M. Zhang, and Y.-Z. Zhang, "Cosmological evolution of a quintom model of dark energy," Physics Letters B, vol. 608, no. 3-4, pp. 177-182, 2005.

[52] R. Lazkoz and G. León, "Quintom cosmologies admitting either tracking or phantom attractors," Physics Letters B, vol. 638, no. 4, pp. 303-309, 2006.

[53] Z.-K. Guo, Y.-S. Piao, X. Zhang, and Y.-Z. Zhang, “Two-field quintom models in the $w-w^{\prime}$ plane," Physical Review D, vol. 74, no. 12, Article ID 127304, 2006.

[54] R. Lazkoz, G. León, and I. Quiros, "Quintom cosmologies with arbitrary potentials," Physics Letters B, vol. 649, no. 2-3, pp. 103110, 2007.

[55] M. Alimohammadi, "Asymptotic behavior of $\omega$ in general quintom model," General Relativity and Gravitation, vol. 40, no. 1, pp. 107-115, 2008.

[56] M. R. Setare and E. N. Saridakis, "Coupled oscillators as models of quintom dark energy," Physics Letters B, vol. 668, no. 3, pp. 177-181, 2008.

[57] M. R. Setare and E. N. Saridakis, "Quintom cosmology with general potentials," International Journal of Modern Physics D, vol. 18, no. 4, pp. 549-557, 2009.

[58] M. R. Setare and E. N. Saridakis, "Quintom model with $\mathrm{O}(N)$ symmetry," Journal of Cosmology and Astroparticle Physics, vol. 2008, no. 9, article 026, 2008.

[59] M. R. Setare and E. N. Saridakis, "Quintom dark energy models with nearly flat potentials," Physical Review D, vol. 79, no. 4, Article ID 043005, 2009.

[60] G. Leon, R. Cardenas, and J. L. Morales, "Equilibrium sets in quintom cosmologies: the past asymptoticdynamics," http:// arxiv.org/abs/0812.0830.

[61] A. De Felice and S. Tsujikawa, " $f(R)$ theories," Living Reviews in Relativity, vol. 13, p. 3, 2010.

[62] T. P. Sotiriou and V. Faraoni, “ $f(R)$ theories of gravity," Reviews of Modern Physics, vol. 82, no. 1, pp. 451-497, 2010. 
[63] S. Tsujikawa, "Modified gravity models of dark energy," in Lectures on Cosmology, vol. 800 of Lecture Notes in Physics, pp. 99-145, Springer, 2010.

[64] G. N. Remmen and S. M. Carroll, "Attractor solutions in scalarfield cosmology," Physical Review D, vol. 88, no. 8, Article ID 083518, 2013.

[65] Z.-K. Guo, Y.-S. Piao, R.-G. Cai, and Y.-Z. Zhang, "Inflationary attractor from tachyonic matter," Physical Review D, vol. 68, no. 4, Article ID 043508, 2003.

[66] L. A. Ureña-López and M. J. Reyes-Ibarra, "On the dynamics of a quadratic scalar field potential," International Journal of Modern Physics D, vol. 18, no. 4, pp. 621-634, 2009.

[67] V. A. Belinsky, L. P. Grishchuk, I. M. Khalatnikov, and Y. B. Zeldovich, "Inflationary stages in cosmological models with a scalar field," Physics Letters B, vol. 155, no. 4, pp. 232-236, 1985.

[68] T. Piran and R. M. Williams, "Inflation in universes with a massive scalar field," Physics Letters B, vol. 163, no. 5-6, pp. 331335, 1985.

[69] B. Ratra and P. J. E. Peebles, "Cosmological consequences of a rolling homogeneous scalar field," Physical Review D, vol. 37, no. 12, pp. 3406-3427, 1988.

[70] A. R. Liddle and D. H. Lyth, Cosmological Inflation and LargeScale Structure, Cambridge University Press, Cambridge, UK, 2000.

[71] V. V. Kiselev and S. A. Timofeev, "Quasiattractor dynamics of $\lambda \phi^{4}$-inflation," http://arxiv.org/abs/0801.2453.

[72] S. Downes, B. Dutta, and K. Sinha, "Attractors, universality, and inflation," Physical Review D, vol. 86, no. 10, Article ID 103509, 14 pages, 2012.

[73] J. Khoury and P. J. Steinhardt, "Generating scale-invariant perturbations from rapidly-evolving equation of state," Physical Review D, vol. 83, no. 12, Article ID 123502, 2011.

[74] S. Clesse, C. Ringeval, and J. Rocher, "Fractal initial conditions and natural parameter values in hybrid inflation," Physical Review D, vol. 80, no. 12, Article ID 123534, 2009.

[75] V. V. Kiselev and S. A. Timofeev, "Quasiattractor in models of new and chaotic inflation," General Relativity and Gravitation, vol. 42, no. 1, pp. 183-197, 2010.

[76] G. F. R. Ellis, R. Maartens, and M. A. H. MacCallum, Relativistic Cosmology, Cambridge University Press, Cambridge, UK, 2012.

[77] Y. Wang, J. M. Kratochvil, A. Linde, and M. Shmakova, "Current observational constraints on cosmic doomsday," Journal of Cosmology and Astroparticle Physics, vol. 2004, no. 12, article 006, 2004. 

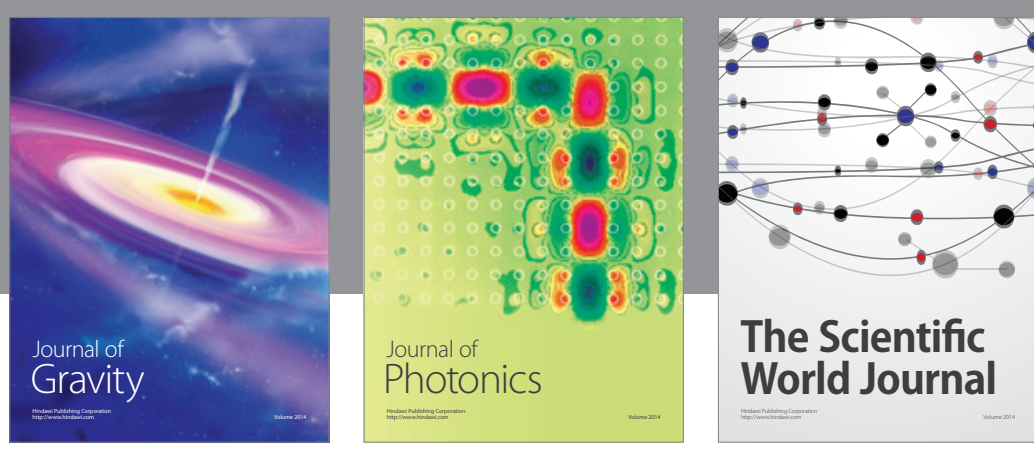

The Scientific World Journal
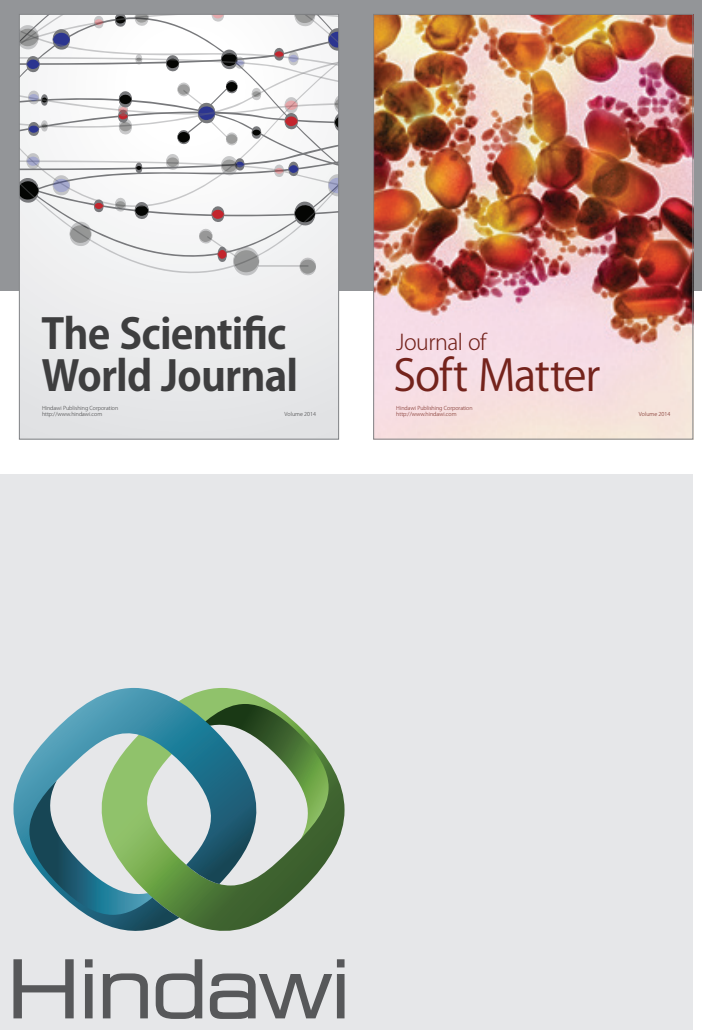

Submit your manuscripts at

http://www.hindawi.com

nternational Journal of

Statistical Mechanics
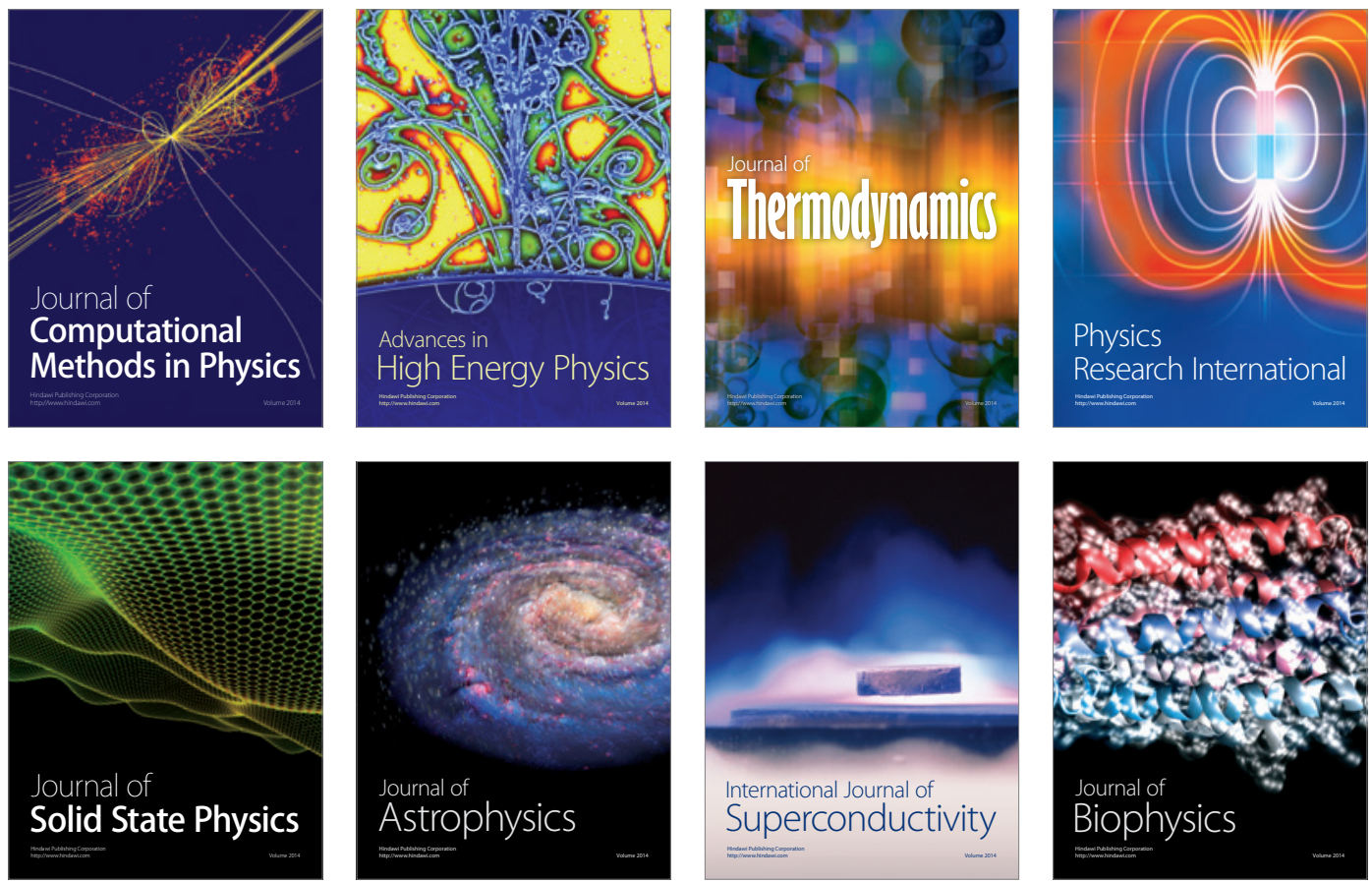
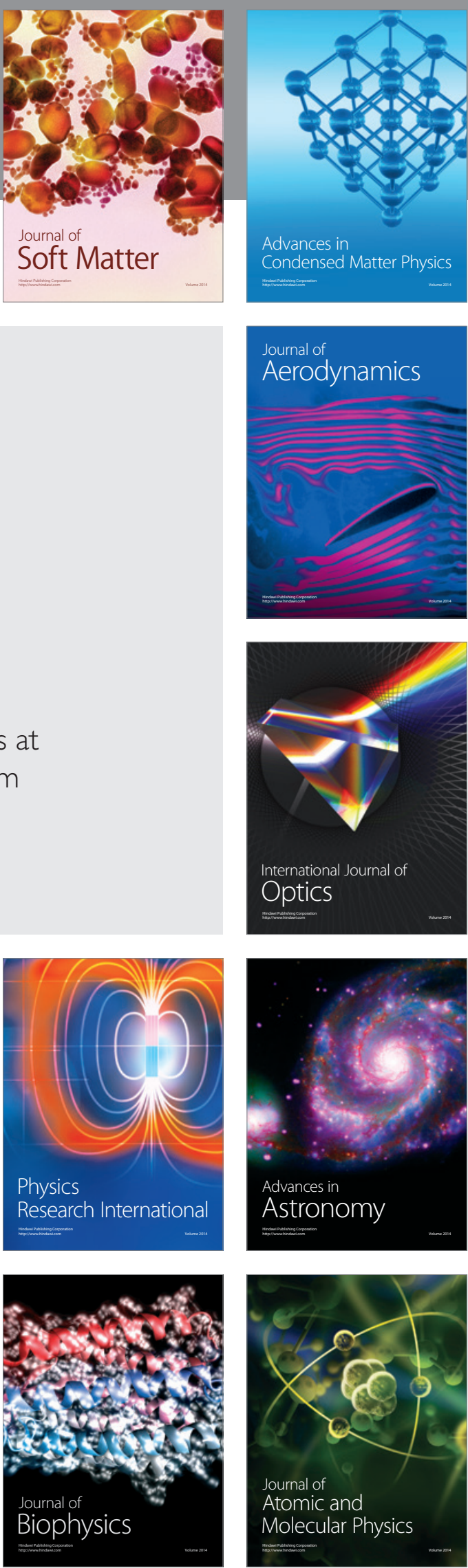